\title{
Braucht eine Textlinguistik Kategorien des Sinns? Sinnkritische Bemerkungen zu Frege, Coseriu und Luhmann
}

\author{
Karsten Hvidtfelt Nielsen \\ Institut für Sprache, Literatur und Kultur, Abteilung für Deutsch, Universität \\ Aarhus, Dänemark
}

In this paper, I inquire whether the discipline of discourse analysis (textual linguistics) needs to accept categories of meaning into its work. I direct the inquiry from the position of meaning scepticism such as argued in Nielsen (2003). The consequences of discarding meaning as an object of textual research are illuminated by means of a model that, taking textual usage for its object, turns usage into a purely material, deterministic and decidable affair. As contrast, three non-material, non-deterministic and undecidable models of meanings are presented: one assembling Frege's scattered comments on linguistic matters into a conception of meaning as sceptical as my own, one depicting Coseriu's approach to meaning as a hermeneutic mix of psychology (Bühler) and glossematics (Hjelmslev), and one attempting to bring out the internal (and intended) paradoxes of Luhmann's constructivistic theory of meaning. I conclude by intimating that discourse analysts will have to choose: either they abandon meaning, or their work will have an equal share in the undecidabilities (be they hermeneutic, constructivistic or whatever) of meaning.

\section{Captatio benevolentiae}

Die Textlinguistik gibt es nicht. So wenig wie ihr Mutterboden, die Linguistik, sich heute als eine methodisch und theoretisch festgefügte Einheit beackern lässt, so wenig präsentiert sich ihr Sprössling als ein einheitliches Wissensgebiet. Unter Textlinguistik firmiert eine reiche Varietät von Disziplinen, die kaum mehr als der Wunsch nach Erschließung der textuellen Dimensionen des sprachlichen Universums zusammenhältt ${ }^{1}$. Gegen diese Vielfalt ist natürlich nichts einzuwenden; lässt diese doch sich als das erfreuliche Zeichen sowohl der Fruchtbarkeit textbezogener Fragestellungen als auch der Diversität der in Angriff genommenen Phänomene mühelos interpretieren. Bei diesem wahren embarras de richesse scheint das Gebot der Stunde eher Arbeit an schon vorhandenen Ansätzen zu sein als deren Anzahl um noch eine Variante erhöhen zu wollen. Nichtsdestoweniger trifft Letzteres gerade die Absicht des vorliegenden Aufsatzes: der textlinguistisch orientierten Fachwelt einen neuen Approach $\mathrm{zu}$ dem Studium textueller Phänomene vorzulegen.

Es erhellt von selber, dass bei der reichen Tradition, die textlinguistische Disziplinen ihrer kurzen Dauer zum Trotz aufzuweisen vermögen, kein Neuansatz mit dem 
Anspruch auf vollständige Originalität auftreten kann - oder sollte. Mit existierenden Forschungssträngen durchweg brechen $\mathrm{zu}$ wollen ist meistens ein Verfahren von zweifelhaftem Wert. Wie unten relativ ausführlich darzulegen sein wird, verdankt der hier zu vertretende Approach einer Reihe vorhergehender Einsichten und Ergebnisse entscheidende Impulse für die eigenen Fragestellungen. Wenn ich aber bei allen fachlichen Abhängigkeiten dennoch der Ansicht bin, mit meinem Vorschlag zu einer neuen Textlinguistik von der Tradition in einigen Punkten radikal abzuweichen, dann bin ich mir durchaus der Misslichkeiten eines solchen Verfahrens voll bewusst. Die Radikalität meines Ansatzes mag bei allem Überfluss, der die heutige Forschungslage der textlinguistischen Disziplinen prägt, die Errichtung noch einer Spezies vielleicht rechtfertigen; sie weckt aber den ebenso berechtigten Verdacht, dabei werde eher aus Unkenntnis existierender Forschung fabuliert, als dass die vorgeschlagene Neuerung einen bisher versäumten Forschungsweg eröffnen sollte.

Für die Ungewöhnlichkeit der hier vorgeführten Thesen soll also zunächst entschuldigt werden. Der knappe Raum eines Aufsatzes wird selbstverständlich nicht in jeder Hinsicht mit allen Zweifeln an der Haltbarkeit oder gar der Angemessenheit des Vorgeschlagenen aufräumen können. In seinen Grundeinstellungen geht der hier unterbreitete Ansatz auf sprachlogische Fragestellungen zurück, denen ich in meiner Abhandlung Interpreting Spinoza's Arguments ausführlich nachgegangen bin $^{2}$. Auf diese werde ich mich gelegentlich berufen. In der Hauptsache aber gedenke ich meine Vorstellungen und Argumente so vorzuführen, dass ihnen auch ohne besondere Vorkenntnisse aus dem Bereich der analytischen Sprachlogik zu folgen sein wird. Es geht mir nicht darum, unter dem Schleier eines textlinguistischen Vorwandes ein kurzgefasstes Resümee des schon Publizierten zu präsentieren, sondern gewonnene Einsichten für die Erforschung textueller Phänomene fruchtbar zu machen. Deshalb sind auch die Terminologie und die Begrifflichkeit, die ich hier verwende, der logischen Prägung entkleidet und den für Neologismen ohnehin recht aufgeschlossenen Usancen der textlinguistischen Disziplinen weitgehend angepasst. Mit diesem Aufsatz soll ein genereller Rahmen für textlinguistische Arbeit abgesteckt werden. In einer späteren Publikation gedenke ich den Rahmen mit einem konkreten Forschungsprojekt zu füllen.

\section{DAS MOdeLL}

\subsection{Drei Forderungen}

Ontologisch, wenn ich so frei sein darf, vertrete ich eine Position, für die ich den Namen epistemischer Monismus geprägt habe ${ }^{3}$. Sicher mag es andere Welten, andere Phänomenbereiche als die der physischen Realität geben; wenn aber solche wissenschaftlich erforscht werden sollen, reduzieren sie sich auf ihr materielles Substrat. So der Hauptgedanke meiner Spezies des Monismus. Ich bin der Auffassung mit dieser Position einen von Spinozas berühmtesten Leitsätzen aus der Ethica zu vertreten, ja eigentlich damit die Grundhaltung seiner ganzen Epistemologie auf einen einfachen Nenner gebracht zu haben ${ }^{4}$, bin aber selbstverständlich bereit dafür die alleinige Verantwortung zu übernehmen. 
Karsten Hvidtfelt Nielsen, Braucht eine Textlinguistik Kategorien des Sinns?

Wie dem auch sei: Aus der Position des epistemischen Monismus folgt mühelos die erste Forderung, die ich mit diesem Ansatz aufstellen möchte: Reduktion des zu Erforschenden auf Materialität, auf physis. Nur die physische Wirklichkeit (einschließlich ihrer biologischen, neurologischen, elektronischen etc. Erscheinungsformen) kommt als Gegenstandsbereich für den hier versuchten Approach in Frage. Es mag sich vieles andere ereignen, wenn Texte von Sprachgebrauchern prozessiert (erstellt/verstanden) werden. Derlei aber zu betrachten erübrigt sich in dem Maße, als sich dessen Erforschung wissenschaftlichen Standards nähert. Damit ist natürlich auch gesagt, dass die Textlinguistik, der ich das Wort rede, jeden prinzipiellen Unterschied zwischen den Praktiken und Fragestellungen der Naturwissenschaften und ihren eigenen Vorgehensweisen ablehnt. Dies soll nicht heißen, dass Textlinguisten ihre Arbeit einem Team von Schallphysikern, Physiologen und Neurologen überlassen sollen. Ganz im Gegenteil. Wohl werden Sprachwissenschaftler meiner Meinung nach gut beraten sein, die eigene Forschungstradition, ihre Begriffe und ihre Argumente, im Lichte dessen, was in der Naturwissenschaft als Theorie und Überprüfung von Theorien gilt, noch einmal zu überdenken. Aber die Fragestellungen, mit denen sich die textlinguistischen Disziplinen bisher beschäftigt haben, werden auch nach solcher Revision das ureigene Feld einer sprachwissenschaftlich ausgerichteten Textlinguistik bleiben.

Mit der Reduktion des Forschungsbereichs auf Materielles geht auch die zweite Forderung einher. Diese hat eher die Form einer Arbeitshypothese. Es soll angenommen werden, dass die Phänomene, die eine sich auf das Physische reduzierende Textlinguistik untersucht, sich nach deterministischen Prinzipien ereignen. Mit diesem Bekenntnis zu Determinismus ist weniger eine philosophische Haltung anvisiert, die es zunächst zu erklären und zu verteidigen gäbe ${ }^{5}$, als eine Angleichung an die Selbstverständlichkeit, mit der deterministische Annahmen und Vorstellungen die naturwissenschaftlichen Forschungsgänge weitgehend begleiten und bestimmen ${ }^{6}$. Was unter und in Sprachgebrauchern, wenn textlinguistische Phänomene sich ereignen, vor sich geht, soll also als ein deterministisches Verfahren untersucht werden.

Die Arbeitshypothese mag natürlich falsch sein. Die textlinguistische Wirklichkeit mag auch dem Deterministen einige unerwartete Überraschungen bescheren. Andere Forschungszweige stehen schon seit einiger Zeit vor einem ähnlichen Dilemma. Reichen die Praktiken der heutigen Naturwissenschaft für die Erforschung ihrer jeweiligen Gebiete aus, oder müssen alternative Strategien und neue Methoden entwickelt werden, um einer Wirklichkeit gerecht zu werden, die sich dem deterministischen Zugang schlecht zu fügen scheint? Die Medizin wäre zu erwähnen, oder die Bewusstseinsforschung, in der seit einigen Jahren die Debatte darüber geführt wird, wie einem Phänomen wie dem menschlichen Geist wissenschaftlich beizukommen ist ${ }^{7}$.

Die textlinguistischen Disziplinen aber sind von anderen Forschungstraditionen und anderen Fragestellungen geprägt. Sie sind in einem wissenschaftlichen Milieu groß geworden, in dem die Forderung nach deskriptivem Verfahren und Vermögen das Forschungsfeld mit einer solchen Ausschließlichkeit beherrscht hat, dass Fragen, die 
Karsten Hvidtfelt Nielsen, Braucht eine Textlinguistik Kategorien des Sinns?

auf kausale Zusammenhänge und deren Erklärungen abzielten, gar nicht aufkommen konnten. Seit dem Ausgang des Positivismus ist Determinismus in - fast - allen linguistischen Disziplinen Anathema geworden. Auf die isolierte Ausnahme wird unten stellenweise einzugehen sein. Hier bleibt zunächst festzuhalten, dass der Determinismus eine noch nicht probierte Hypothese in textlinguistischen Arbeitsgängen darstellt ${ }^{9}$. Es ist eine offene Frage, ob der Determinismus ausreichen wird, um in allen Bereichen die wissenschaftliche Neugier voll $\mathrm{zu}$ befriedigen. Persönlich meine ich, dass Alternativstrategien, ihrer gegenwärtigen Beliebtheit zum Trotz, alle am Ende von dem monistischen Determinismus, der das Gros der wissenschaftlichen Arbeit noch bestimmt, wieder eingefangen werden. Aber diese Meinung ist für die weitere Argumentation im Grunde gleichgültig.

Das Feld, das es in diesem Abschnitt zu modellieren gilt, soll also als ein rein materielles und ein rein deterministisches konzipiert werden. Ich halte die erste Option für eine Sache der Ontologie, die zweite für eine Arbeitshypothese. Mit der dritten, und letzten, Forderung betreten wir den Bereich der Verifikation ${ }^{10}$. Es soll hier nicht versucht werden, der gar nicht leichten Frage danach nachzugehen, wie textlinguistische Forscher über die empirische Haltbarkeit ihrer Thesen und Theorien entscheiden. Natürlich hat das Fach bestimmte Routinen dafür entwickelt, die es ihm erlauben auf kompetente Art und Weise in den meisten Situationen über die empirische Tragfähigkeit seiner Begriffe zu befinden. Vielleicht darf man in grober Verkürzung solche Routinen auf den Nenner der Nachvollziehbarkeit bringen. Die Befunde der textlinguistischen Forschung sollen für Fachleute nachvollziehbar sein. Wie gesagt, die Komponenten dieser Fähigkeit zu bestimmen und herauszubringen ist ein Diffiziles. Fachliche Erfahrung, Handhabe bestimmter Tests und Vergleiche, Wissen um geeignete Gegenbeispiele, geschulte sprachliche Intuition, logisches Vermögen, Sinn für begriffliche Distinktionen, Beherrschung der relevanten Fachliteratur und vieles andere bestimmen die Nachvollziehbarkeit einer textlinguistischen Argumentation. Aber eines darf als sicher gelten. Nachvollziehbarkeit reicht nicht an die Eigenschaft heran, die man mit einem Ausdruck aus der Logik Entscheidbarkeit nennen kann.

Unter Entscheidbarkeit versteht man die Fähigkeit, die Frage nach der Subsumption eines Phänomens unter einem gegebenen Begriff, oder nach dessen Zugehörigkeit zu einer gegebenen Menge nach rein mechanischen Prinzipien mit Ja oder Nein zu beantworten ${ }^{11}$. In der praktischen Arbeit der Naturwissenschaft kommt Entscheidbarkeit meistens in Verbindung mit Experimenten zum Tragen. Das Experiment, ein rein Mechanisches, soll den Wissenschaftlern helfen, die empirische Haltbarkeit ihrer Theorien zu testen. Im Idealfall ist ein Experiment so zu gestalten, dass es auf präzise definierte Fragen ein Ja oder ein Nein ermöglicht. In dieser Forschungswelt gelten Theorien als Prognosen, die sich über Experimente bewähren müssen. So nicht die Theorien und Begriffe in dem textlinguistischen Forschungsmilieu. Auch sie müssen sich selbstverständlich bestimmten Routinen und Überprüfungsstrategien unterwerfen lassen, aber von Prognosen, über deren Bestand experimentell zu entscheiden wäre, wird man wohl in textlinguistischen Zusammenhängen kaum sprechen ${ }^{12}$. 
Karsten Hvidtfelt Nielsen, Braucht eine Textlinguistik Kategorien des Sinns?

Das Modell, von dem gleich die Rede sein soll, soll den beiden ersten Forderungen in vollem Maße gerecht werden. Mit der dritten Forderung verhält die Sache sich anders. Was man genauer in einer materiellen und deterministischen Textlinguistik sich unter Entscheidbarkeit vorzustellen hat, und wie die herkömmliche Nachvollziehbarkeit des Faches in die geforderte Entscheidbarkeit zu überführen wäre, lässt sich am besten anhand eines konkreten Forschungsprojekts erklären und nachweisen. Da ich in Kürze ein solches vorzulegen gedenke, soll auf eine weitere Explikation dieses Punktes verzichtet werden. Unten wird in Verbindung mit der Diskussion der Sinnfrage wieder auf die Problematik der Entscheidbarkeit zurückzukommen sein. Aber eine befriedigende Erklärung wird auch am Ende dieses Aufsatzes ausstehen.

\subsection{Das Modell}

Mit Modell wird hier keine mengentheoretische Konstruktion gemeint. Es soll gelegentlich auf mathematische Formalisierungen zurückgegriffen werden. Solche Ansätze zur Mathematisierung haben aber keinen Selbstzweck, sondern dienen ausschließlich der Übersichtlichkeit der Darstellung. Das Modell, das ich darbiete, stellt, wie unter Textlinguisten üblich, eine idealisierte Repräsentation von demjenigen Weltausschnitt dar, mit dem die hier anvisierte Textlinguistik sich zu beschäftigen vorgenommen hat. Jedes Modell unterwirft die empirische Wirklichkeit einer doppelten Reduktion: (a) aus der Menge der möglichen Ausschnitte wird einen spezifischen ausgewählt; (b) aus der konkreten Vielfalt dessen, was im ausgewählten Ausschnitt zu berücksichtigen wäre, wird eine idealisierte Vereinfachung abstrahiert. Ein Modell aufstellen heißt demnach eine besondere Forschungsperspektive anlegen. Um welche es hier gehen wird, ist schon angedeutet worden. Für unser Modell kommen nur materielle Vorgänge in Frage. Aber auch diese sind natürlich in solcher Fülle vorhanden, dass eine weitere reduktive Idealisierung notwendig ist.

Keine Abstraktion ist begriffsneutral. Für die folgende Modellierung haben Begriffe aus dem computationellen Funktionalismus sowie aus dem Sprachbehaviorismus Pate gestanden ${ }^{13}$. Damit soll kein Streit über deren Meriten und bisherigen Leumund in der Sprachwissenschaft vom Zaun gebrochen werden ${ }^{14}$. Das Modell will natürlich Chomskyaner und Kognitivisten wenig ansprechen. Aber umgekehrt enthält es nichts, dem jemand, meines Wissens, ernsthaft würde widersprechen wollen. Streit entstünde eher bei dem, was das Modell auslässt.

In einer ersten Annäherung lässt sich der hier in Betracht kommende Weltausschnitt als die materielle Realität der Textverwendung charakterisieren. Statt Textverwendung kann man auch Sprachverwendung sagen. Nur hat der erstere Terminus den Vorteil deutlicher als der letztere darauf hinzuweisen, dass das, was verwendet wird, den Forscher nur als konkrete Materialität interessiert. Ein Text ist ein Stück Materialität. In wie vielen Formen und Schattierungen materielle Texte vorkommen können, ist in jeder zweiten Einführung in die Textlinguistik zu finden. Ihre Erörterung soll deshalb hier übersprungen werden. Materielle Texte gehören also zum Forschungsbereich einer 
deterministischen Textlinguistik, erschöpfen ihn aber keineswegs. Die hier dargebotene Textlinguistik ist eine von der Textverwendung, nicht nur vom Text.

$\mathrm{Zu}$ der Textverwendung gehören außer Texten auch Textverwender. Darunter sind, wiederum recht banal, solche Entitäten, die Texte prozessieren, zu verstehen. Wir werden uns wenig darum kümmern, ob diese Textprozessoren chemisch-biologisch, wie Menschen, elektronisch-physisch, wie Computer, oder anderswie realisiert sind. Natürlich interessiert uns an erster Stelle die menschliche Textverwendung; nur wollen wir dieser keine Ausschließlichkeit zusprechen. Prozessieren lassen sich Texte auf zwei unterschiedliche Weisen: sie werden entweder produziert oder rezipiert, erstellt oder verstanden. Auch dieser Unterschied ist der textlinguistischen Fachwelt zu bekannt, als dass es nötig wäre, die dazugehörigen Begrifflichkeiten noch einmal an dieser Stelle zu exerzieren. Schließlich gehört zu dem Bereich diejenige Varietät von Übermittlungskanälen, über die Texte vom Produzenten zum Rezipienten gelangen.

Produzieren und Rezipieren sind Aktivitäten, die jeder Textverwender wechselweise übernehmen kann. Diese Rollen sind natürlich in die vorhergehenden Prozesse des Spracherwerbs integriert ${ }^{15}$. Wie bei der Erlernung einer Sprache (des Sprechens) spielen auch bei der Konditionierung zu Textverwendung das sich Einstellen auf Erfolgskriterien eine entscheidende Rolle ${ }^{16}$. Wie solche auf die Textaktivitäten der Sprecher einwirken, soll hier nicht weiter diskutiert werden. Nur ist daran festzuhalten, dass jedem Textprozessieren (von dem schwer auffindbaren ersten abgesehen) eine Reihe von früheren vorausgegangen ist. Im Folgenden soll nur von erfahrenen Textprozessoren die Rede sein. Mit dieser Einschränkung gilt: jedes Textprozessieren hat eine Vergangenheit.

Der Textprozessor $\mathrm{T}$ besitzt ein Steuerungsaggregat $\mathrm{S}$ und ein damit verbundenes Interface I mit der Umwelt. Über I gehen efferente Aktivitäten von $\mathrm{T}$ aus, und über I werden afferente Aktivitäten T zugeführt. Der Einfachheit halber nehmen wir an, dass alle Aktivitäten, afferent wie efferent, $S$ in Mitleidenschaft ziehen. Efferente Aktivitäten starten in S, afferente enden in S.

Ich bringe jetzt meine Arbeitshypothese von Determinismus in Anschlag. Zu jedem Zeitpunkt $t$ befindet $S$ sich in einem bestimmten Zustand $s_{t}$. Der Übergang von einem Zustand zum nächsten wird von einer Aktivität, afferent oder efferent, mit verursacht (von rein internen Aktivitäten wird hier abgesehen). Sei $s_{t}$ ein Zustand, dann heiße die afferente Aktivität, die $s_{t}$ unmittelbar vorangeht, $a_{t^{\prime}}$ und die efferente Aktivität, die unmittelbar auf $s_{t}$ folgt, $e_{t}$. Seien $s_{t}$ und $s_{t+1}$ zwei aufeinanderfolgende Zustände des Steuerungsaggregats, dann gelten folgende Determinationsverhältnisse (Determination wird mit $\rightarrow^{\prime}$ angegeben; mit , $+{ }^{\prime}$ wird Verdoppelung der determinierenden Faktoren angegeben): $a_{t}+s_{t} \rightarrow s_{t+1}, s_{t} \rightarrow e_{t}, s_{t}+e_{t} \rightarrow s_{t+1}$. Jeder Zustand des Steuerungsaggregates verursacht zusammen mit entweder der unmittelbar vorangehenden afferenten oder der unmittelbar folgenden efferenten Aktivität den nächsten Zustand des Steuerungsaggregates. Bei jeder Aktivität tritt also das Steuerungsaggregat $S$ in einen neuen Zustand über. Der neue Zustand mag, aber muss nicht vom vorigen verschieden 
Karsten Hvidtfelt Nielsen, Braucht eine Textlinguistik Kategorien des Sinns?

sein ${ }^{17}$. Betrachten wir das Tripel $\left\langle\mathrm{s}_{\mathrm{t}}, \mathrm{e}_{\mathrm{t}} / \mathrm{a}_{\mathrm{t}} \mathrm{s}_{\mathrm{t}+1}>\right.$ als eine Repräsentation von dem Übergang von einem Zustand zum nächsten im Steuerungsaggregat $S$, dann können wir das Benehmen von $\mathrm{S}$ im Intervall $<\mathrm{t}, \mathrm{t}+\mathrm{n}>$ mit dem n-Tupel $<<\mathrm{s}_{\mathrm{t}}, \mathrm{e}_{\mathrm{t}} / \mathrm{a}_{\mathrm{t}}, \mathrm{s}_{\mathrm{t}+1}><\mathrm{s}_{\mathrm{t}+1}, \mathrm{e}_{\mathrm{t}+1} / \mathrm{a}_{\mathrm{t}+1}, \mathrm{~s}_{\mathrm{t}+2}>, \ldots$ $,<\mathrm{s}_{\mathrm{t}+\mathrm{n}-1}, \mathrm{e}_{\mathrm{t}+\mathrm{n}-1} / \mathrm{a}_{\mathrm{t}+\mathrm{n}-1}, \mathrm{~s}_{\mathrm{t}+\mathrm{n}}>>$ wiedergeben.

$\mathrm{Zu}$ jedem Zeitpunkt ist also der Zustand eines Steuerungsaggregats durch seine unmittelbare Vergangenheit konditioniert. Da aber diese wiederum von seinem unmittelbaren Vorgänger konditioniert, diese wiederum von dem, was ihm vorherging, und so weiter, bewahrt jeder Zustand durch seine jeweilige Konditionierung eine ,Erinnerung' an frühere Zustände. In den meisten Fällen wird die ,Erinnerung' an, sagen wir, den Zustand $s_{t-n}$ zum Zeitpunkt t durch Zustandsänderungen zwischen t-n und t erhebliche Modifikationen erlitten haben. Nur in seltenen Fällen wird ein Zustand mehrere Aktivitäten als derselbe überleben können.

Textprozessoren interagieren mit einer Umwelt, die auch andere Textprozessoren einschließt. Insofern als die efferenten Aktivitäten der einen über Interface und Umwelt zu afferenten Aktivitäten der anderen werden und vice versa, erfolgen Effekte im Benehmen der Steuerungsaggregate, deren Gesamtwirkung man als Koordination bezeichnen kann. Jeder Textprozessor bestimmt durch sein Verhalten Momente im Verhalten der anderen mit und wird selber in seinem Verhalten davon mit bestimmt, wie diese sich verhalten. Wird im geeigneten Umfeld dem Prozess der Koordination zwischen Textprozessoren eine genügende Laufzeit mit stabiler und dichter Frequenz der Koordinationshandlungen gegeben, entstehen komplexe Verhaltensformen zwischen den koordinierten Einheiten. Jene sind natürlich in erster Linie in den umfassenderen Prozess der sozialen Verhaltenskoordination integriert, aber auch die davon abhängige Textverwendung läst sich begreifen als das sich ständig modifizierende Ergebnis der verhaltensbedingten Koordination zwischen Textprozessoren ${ }^{18}$.

In diesem Koordinationsprozess spielen die physischen Texte eine unabdingbare Rolle. Ohne die käme es selbstredend zu keiner Koordination zwischen den Textprozessoren. Texte entstehen über Zeit und werden über Zeit rezipiert. Produktion sowie auch Rezeption mögen kontinuierlich oder mit Unterbrechungen verlaufen. Texte verlassen das Interface des (oder der) Textproduzenten und erreichen mit zeitlicher Verzögerung unterschiedlichster Zeitdauer die jeweiligen Interfaces der Rezipienten.

Wollte man nun die tatsächliche Arbeit zwischen koordinierten (z. B. menschlichen) Textprozessoren in unserem Modell darstellen, geriete man schnell in Schwierigkeiten. Schon beim Versuch das Verhältnis zwischen einem Textproduzenten und einem bestimmten von ihm produzierten Text zu modellieren, stieße das Modell schnell ins Unübersichtliche. Auch wenn man sich über eine passende Einteilung des Textes einigen könnte (sei es nur die von Satz zu Satz), müsste das Modell diejenigen efferenten Aktivitäten, die für jede Textstelle zuständig waren (die über das Interface dafür Ursache waren), mit dieser verbinden. Afferente Aktivitäten wären vermutlich auch zu berücksichtigen. Die produktive Arbeit am Text mag durchaus Intervalle einschließen, in denen der Produzent sich in einen Rezipienten des eben oder früher Produzierten verwandelt. 
Karsten Hvidtfelt Nielsen, Braucht eine Textlinguistik Kategorien des Sinns?

Wenn der Wert eines Modells von praktischem Nutzen abhängig ist, den es der empirischen Erforschung eines Gegenstandsbereichs bringt, stünde es daher schlimm um das hier vorgeschlagene Modell. Konkrete Arbeit zu leiten ist aber nicht seine Aufgabe. Wie oben angedeutet soll erst in einem späteren Schritt ein eigentliches Forschungsprojekt definiert werden. Das Modell dient zunächst als Konkretisierung der Forderungen, die einem solchen Projekt vorausgehen. Ein Modell, das wie das hier angedeutete die Textverwendung als eine materielle und deterministische Verhaltensart repräsentiert, soll im Folgenden ein MD-Modell genannt werden.

\section{Modelle des Sinns}

\subsection{Zum Begriff des Sinnes}

Für das, was die Textlinguistin wohl am nachhaltigsten in einem MD-Modell vermisst, gibt es viele verschiedene Namen. Es lässt sich darüber streiten, ob solche terminologische Vielfalt einer phänomenalen Tatsächlichkeit entspricht, oder ob sie ihre Diversifikation einer gewissen Beliebigkeit oder sogar Unentscheidbarkeit des zu bezeichnenden Phänomens verdankt. Wer den in der Sprach- und Textverwendung messbaren Impulsen einen Zeichencharakter zuschreibt, steht vor dem Problem, für ein nicht messbares, ein nicht beobachtbares Etwas irgendeine über- oder neben-physische Realität beanspruchen zu müssen ${ }^{19}$.

Als Zeichen scheinen Texte (Worte) schon vom Anfang des europäischen Sprachdenkens an gegolten zu haben. Hier ist nicht der Ort, diese Geschichte auch nur skizzenhaft andeuten zu wollen ${ }^{20}$. Das längste und für das heutige Sprachbild ${ }^{21}$ vielleicht folgenreichste Kapitel der europäischen Textvergangenheit macht das bemühte Nachdenken über den Status der Heiligen Schriften, die sacra hermeneutica, aus. Darauf soll nur hingewiesen werden, weil diese lange Zeit uns die Begrifflichkeit des Text- oder Schriftsinnes beschert hat. Die christlichen Exegeten mochten in der Anzahl und der Bestimmung der Auslegungsmöglichkeiten der Heiligen Schriften uneinig sein. Jeder Streit aber fußte auf der als selbstverständlich hingenommenen Überzeugung, die Schrift habe Sinn. Schriften, Texte sind sinnvolle Gebilde. Wie ein Text etwas haben oder von etwas voll sein konnte, was selbst keine physische Realität besitzt, war den christlichen Exegeten mit dem den Text verursachenden Prinzip (Gott) als eine Selbstverständlichkeit mitgegeben, sollte aber ihren säkularen Nachfolgern sehr unterschiedliche Denkanstrengungen abzwingen.

Von der behavioristischen Zeichentheorie abgesehen ${ }^{22}$ gelten Zeichen als mindestens zweiwertig. Sie haben eine materielle Seite und eine oder mehrere andere, die ebenso schwer zu benennen wie zu bestimmen ist (sind). Vielleicht neigt der heutige Sprachgebrauch unter Linguisten dazu, für diese anderen Seiten das Wort Inhalt zu verwenden. Dass ich dafür lieber von Sinn sprechen will, mag mit meiner Beschäftigung mit den Christologischen Voraussetzungen des heutigen Sprachverständnisses zusammenhängen, ist aber auch, wie ich gleich zeigen möchte, eine Benennung, für die sich im heutigen Wissensbetrieb durchaus namhafte Vertreter finden lassen. 
Karsten Hvidtfelt Nielsen, Braucht eine Textlinguistik Kategorien des Sinns?

In einem MD-Modell der Textverwendung kommen Texte nur als physische Impulse vor. Sie Zeichen zu nennen käme daher Etikettenschwindel nahe. Was Texten damit abgesprochen wird, nenne ich also der Einfachheit halber Sinn. Das Phänomen von Sinn wird oft als ein Zusammengesetztes konzipiert. Die hermeneutica sacra prägte dafür eine Begrifflichkeit, die man, ein wenig profan, als die Lehre von $n$-fachem Schriftsinn bezeichnen kann, wo n Werte zwischen 2 und 4 (im Ausnahmefall 7) annehmen konnte $^{23}$. So gesehen stellt mein MD-Modell den Versuch dar, eine Theorie von 0-fachem Schriftsinn entwickeln zu wollen.

Im Folgenden sollen über drei Modelle von Sinn einige der Schwierigkeiten, die der Sinnbegriff einer wissenschaftlichen Texttheorie bereitet, kurz dargestellt werden. Die Auswahl erhebt keinen Anspruch auf Vollständigkeit, wohl auf eine gewisse Repräsentativität. Da die Besprechungen auf die Sinnfrage konzentriert sind, werden sie der Komplexität der dargestellten Positionen nicht in anderer Hinsicht gerecht werden können. Mein Zugang ist eher sinnkritisch zu nennen und wird daher ohne Zweifel etwas von der epistemischen Toleranz entbehren, mit der Sinnfragen meistens von Linguisten abgehandelt werden.

\subsection{Frege}

Um Freges Schriften wird seit mehr als 30 Jahren unter Philosophen mit einem Eifer gestritten, der an Heftigkeit und Unversöhnlichkeit den exegetischen Anstrengungen des Mittelalters und der frühen Neuzeit um den Heiligen Text in wenig nachsteht ${ }^{24}$. Wer sich in das philosophische Dickicht um Frege hineinwagt, dem wird allmählich klar, dass die Fachwelt zur Zeit weit davon entfernt ist, sich auf ein einheitliches Bild von Freges Denken einigen zu können. Es soll daher extra betont werden, dass die folgenden Kommentare keinen noch so kleinen Beitrag zum philosophischen Disput um Frege leisten wollen, sondern Frege ausschließlich aus der gewählten Sinnperspektive betrachten.

Unter dieser Optik könnte man versucht sein, Freges Sprachtheorie als eine Lehre von zweifachem Schriftsinn ${ }^{25} \mathrm{zu}$ charakterisieren. Hiermit ist nicht, wie vielleicht $\mathrm{zu}$ erwarten wäre, die in alle linguistischen Übersichtswerke eingegangene Theorie von Sinn und Bedeutung gemeint, sondern eine andere, die dieser berühmten sozusagen vorgeschaltet ist ${ }^{26}$. In der Geschichte der Logik wird Frege oft als überzeugter Bekämpfer des Psychologismus vorgestellt. In der noch zu schreibenden Darstellung von Frege als Sprachtheoretiker wäre er janusköpfiger zu porträtieren. Freges Sprachtheorie ist eine Mischung von psychologischen und anti-psychologischen Komponenten. In dem Bereich des Sprachsinns artikuliert sich Freges Doppelsicht in einer Lehre davon, dass jeder Sprachgebrauch sowohl einen psychologischen als auch einen ,logischen' Sinnfaktor im Sprechenden aktiviert. Den ersteren hat Frege meistens Vorstellung ${ }^{27}$ genannt, den letzteren eben Sinn. Wer Freges Sprachdenken als eine Lehre von Schriftsinn darstellen will, kommt also mit zwei Kategorien aus: Vorstellung und Sinn.

Für sprachtheoretische Fragen war Freges Ausgangspunkt die Sprachpsychologie seiner $Z^{2} i^{28}$. Jene vertrat eine Lehre, nach der jede Art von Sprachsinn als Manifestation 
Karsten Hvidtfelt Nielsen, Braucht eine Textlinguistik Kategorien des Sinns?

psychologischer Regungen, die meistens unter dem Namen Vorstellungen firmierten, zu verstehen war. Dass auch Frege mit dieser Theorie spätestens seit seinen Grundlagen der Arithmetik (1884) verhältnismäßig gut vertraut war, lässt sich leicht nachweisen ${ }^{29}$. Auch die beiden ,kanonischen' Schriften zum Sinn, Über Sinn und Bedeutung (1892) ${ }^{30}$ und Der Gedanke (1918) ${ }^{31}$ sind - aus linguistischer Sicht - ohne diese Folie kaum zu verstehen.

In Über Sinn und Bedeutung kommt Frege zu dem Schluss, dass der Sprecher beim Sprachgebrauch dreierlei realisieren kann: Erstens kann er eine Relation zum Gegenstand etablieren, den er bezeichnen will. Diese Relation, oder genauer, den Gegenstand selber nennt Frege Bedeutung ${ }^{32}$. Zweitens hat jeder Sprachgebraucher eine völlig subjektive Vorstellung, die er mit keinem anderen teilen kann ${ }^{33}$. „[D]azwischen liegt der Sinn, der zwar nicht mehr subjektiv wie die Vorstellung, aber doch nicht der Gegenstand selber ist." (SB, 44).

Was Frege mit Sinn hat bezeichnen wollen, ist eine der strittigsten Fragen der philosophischen Fregeforschung ${ }^{34}$. Die sprachtheoretischen Konsequenzen dieses Begriffs lassen sich aus der späten Schrift, Der Gedanke, einigermaßen eindeutig herausfischen. Der Sprachgebraucher aktiviert Sinne und Vorstellungen auf zwei unterschiedliche Arten: Sinne werden vom Sprecher und Hörer erfasst, Vorstellungen sind innere Erlebnisse, die Sprecher und Hörer haben. Das Haben von Vorstellungen wird von Frege in vier Leitsätzen weiter bestimmt: (a) Vorstellungen sind unsinnlich (G, 40); (b) der Ort, wo Vorstellungen vorkommen, ist das Bewusstsein ( $G, 41)$; (c) Vorstellungen setzen einen Träger voraus, der sie eben haben kann $(G, 41)$; (d) Vorstellungen sind privat: keine zwei Träger haben dieselbe Vorstellung ( $G, 41)$.

Was Erfassen der Sinne impliziert und heißt, wird über den Kontrast zu den Leitsätzen (b) zu (d) extrapoliert: (b') Sinne sind nicht im Bewusstsein des Sprechers zu lokalisieren; (c') Sinne gibt es auch unabhängig von dem Träger (von jedem Erfassen der Sinne); (d') Sinne sind objektiv und werden von jedem, der sie erfasst, auf genau dieselbe Art und Weise gefasst. Nur eines verbindet Sinne mit Vorstellungen: beide Typen des Sprachsinnes sind unsinnlich (a). Damit hat Frege sich zu einer Position gezwungen, die in der Fachliteratur als Platonismus oder Realismus bezeichnet wird ${ }^{35}$ : Sinne „sind weder Dinge der Außenwelt noch Vorstellungen. Ein drittes Reich muss anerkannt werden. Was zu diesem gehört, stimmt mit den Vorstellungen darin überein, dass es nicht mit den Sinnen wahrgenommen werden kann, mit den Dingen aber darin, dass es keines Trägers bedarf, zu dessen Bewusstseinsinhalten es gehört." $(G, 43)$.

Also: Sprachgebraucher realisieren zwei Arten von Sprachsinn: eine allen gemeinsame und objektive (Sinn), eine völlig private und subjektive (Vorstellung). Wenn die erstere realisiert wird, bezieht der Sprachgebraucher (durch sein Erfassen) sich auf Einheiten eines Bereiches, der weder physisch noch psychisch ist. Die letztere Art hat ausschließlich Existenz im psychischen Innenraum des jeweiligen Sprachgebrauchers.

Es folgt aus dem logischen Charakter des Sinnes, dass diese Art des Sprachsinns von den jeweiligen Einzelsprachen unabhängig ist. Zwar hat jeder Ausdruck einer gegebenen Sprache Sinn ${ }^{36}$, aber, wie Frege in seinen beiden ,kanonischen' Aufsätzen argumentiert ${ }^{37}$, 
Karsten Hvidtfelt Nielsen, Braucht eine Textlinguistik Kategorien des Sinns?

Sprachgebraucher einer gegebenen Sprache müssen nicht mit demselben Ausdruck denselben Sinn erfassen. Da aber auch jeder Sprachgebraucher seine eigenen, völlig privaten Vorstellungen hat, ist aus Freges Lehre von zweifachem Schriftsinn zu schließen, dass die Kategorien des Sinnes eigentlich loszulösen sind von dem jeweiligen Sprachmaterial. Ob als psychische Vorstellung oder als logischer Sinn, was mit den sprachlichen Äußerungen gemeint werden kann, hat keine ,innere' Verbindung zur Sprache. Zwar brauchen Menschen Sprache um ihr Meinen und Denken veräußerlichen zu können, aber diese Verbindung ist eher akzidentiell. Dass Frege diese Konsequenz selber gesehen und gezogen hat, davon zeugen späte Aufzeichnungen kurz vor dem Tode Freges: „Es ist kein Widerspruch, Wesen anzunehmen, welche denselben Gedanken [Sinn] wie wir fassen können, ohne dass sie ihn in eine sinnliche Form zu kleiden brauchen. Nun aber, für uns Menschen besteht diese Notwendigkeit.“38.

Damit aber kommt man zu dem vielleicht überraschenden Befund, dass Freges Lehre von zweifachem Schriftsinn aus der eigentlichen Sprachbetrachtung herausfällt. Aus Freges Sicht wäre Sprache daher eher als eine rein auf ihre Materialität (das Sinnliche) zu beschränkende Struktur zu beschreiben. Was wir, die Sprachgebraucher, jener Struktur an Sinn hinzufügen, wird zwar mit der Sprachmaterialität verbunden, gehört ihr aber nicht, wie eine zweite und dritte Seite ihrer Natur, an. Auch mit dieser Konsequenz zeigt sich Frege der Sprachpsychologie seiner eigenen Zeit verpflichtet. Er erweiterte zwar deren rein psychologische Bestimmung des Sinns um eine logische Komponente, hielt aber beide Arten des Sinns von dem, was er Sprache nennen würde, fern. Bei Sprachpsychologen wie Steinthal, Lazarus, Wundt und Paul war Sinn auch keine wesentlich sprachliche Kategorie, sondern eine, für die die Psychologie letztlich zuständig war. Frege pflichtete völlig der These von der psychologischen Natur des Sinns als Vorstellung bei, wollte aber die Psychologie um eine Logik des Sinns ergänzt wissen. In keinem Fall fiel ihm der Gedanke ein, Sinn der Sprache einverleiben zu wollen.

In der Geschichte der Sprachtheorie stellt das 19. Jahrhundert eine beachtenswerte Abweichung von einer langen Tradition des europäischen Sprachdenkens da. Vor Freges Jahrhundert hatte die Idee von der Sprachimmanenz des Sinns mit der Selbstverständlichkeit eines kaum beachteten oder diskutierten Axioms geherrscht. Mit dem Anfang des 20. Jahrhunderts sollte diese Vorstellung in den Modellen der Sprachwissenschaft nun mit aller Nachdrücklichkeit und in voller Explizitheit wieder aufgegriffen werden. Dazwischen liegt eine kurze Periode, wo Sprache versuchsweise als ein Mechanismus konzipiert wurde, der bei psychologischen und/oder logischen Komponenten Anleihe machen musste, um zu einer sinntragenden Größe werden zu können ${ }^{39}$.

Eine Textlinguistik, die auf Frege aufbauen würde, stünde vor erheblichen Schwierigkeiten. Die sollen hier nicht einzeln aufgezählt werden. Wer aber nach einem Modell von 0-fachem Sprach- oder Schriftsinn fahnden wollte, käme bei Frege, wie übrigens im ganzen 19. Jahrhundert, unschwer auf seine Kosten. Darüber hinaus weist Freges Modell mehrere Züge auf, die den MD-Theoretiker interessieren müssten. Für 
Frege, wie für sein Jahrhundert, war Sprache gleich Sprachverwendung. Frege scheint diese Gleichung mit einer solchen Selbstverständlichkeit vorausgesetzt zu haben, dass er darauf nie explizite verwiesen hat. In seine Metasprache ist jene daher kommentarlos eingegangen ${ }^{40}$. Weiter hat Frege, wie schon erwähnt, auf der Unentscheidbarkeit der psychologischen Vorstellungen bestanden. Nach Frege entziehen sie sich mit einer Vollständigkeit, die jede Wissenschaft vom psychologischen Sinn zu einer Illusion macht, der schieren Möglichkeit der Identifikation. Um einen späteren Sinnkritiker zu zitieren: „there is no fact of the matter“41. Was Frege aber dafür völlig akzeptiert, ist die Idee einer Wissenschaft von psychologischen Ursachen. Auch in dieser Hinsicht ganz das Kind seines Jahrhunderts hat Frege keine Schwierigkeit, die Berechtigung einer deterministischen Psychologie zu anerkennen ${ }^{42}$. Nur würde eine solche Wissenschaft weder die ,Inhalte' der so verursachten Vorstellungen bestimmen können, noch wüsste sie ein relevantes Wort über die logische Erkenntnis, die die Sinne garantieren, zu sagen. „Die zum Urteilen ... veranlassenden Ursachen tun dies nach psychologischen Gesetzen ... sie haben überhaupt keine innere Beziehung zur Wahrheit; sie verhalten sich zum Gegensatze von wahr und falsch gleichgültig. ${ }^{43}$. Beidem kann der MDTheoretiker beipflichten. Auch er hält eine Wissenschaft von psychologischem Sinn für illusorisch, und seinen Determinismus sieht er gleichfalls jenseits von ,wahr und falsch' untergebracht. Nur den Glauben des 19. Jahrhunderts an einen außerhalb des Physischen wirksamen Determinismus würde er mit Frege nicht teilen.

Wenn aber Sinn, sei es als psychologischer oder logischer Faktor, vom Fragekreis der Wissenschaft ausgeschlossen wird, wie dann überhaupt das tatsächliche Wirken der Sprache erklären. Das war Freges Anliegen nicht. Ihn interessierten nur die erkenntnisvermittelnden Funktionen der Sprache. Für diese erfand er sich sein drittes Reich der allen Sprachgebrauchern gemeinsamen Sinne. Die auf Frege folgende Sprachwissenschaft rückte diese postulierte Gemeinsamkeit des Sprachsinns von der Logik in die Linguistik zurück. Es fragt sich aber, ob ihre Modellierungen vom Sinn nicht Freges spekulative Ontologie nur mit anderen Mitteln weitergeführt haben.

\subsection{Coseriu}

Mit Frege haben wir uns am Rande dessen, was eine Textlinguistik interessieren könnte, aufgehalten. Mit Coseriu rücken wir nicht nur ins Zentrum einer bis vor kurzem allgemein akzeptierten Linguistik zurück, sondern wir werden uns auch mit einem Forscher beschäftigen dürfen, der seine Textlinguistik explizite als eine Linguistik des Sinns bezeichnet hat ${ }^{44}$. In Bälde soll die Frage gestellt werden, was Coseriu mit Sinn gemeint haben kann, aber zunächst ganz kurz zu den Voraussetzungen seiner Textlinguistik.

Coserius Sprachdenken schöpft aus zwei Quellen: dem Strukturalismus und der Sprachpsychologie. Der Erstere scheint Coseriu besonders in der ausgereiften Form der Glossematik angesprochen $\mathrm{zu}$ haben; die Letztere hat Coseriu vorwiegend über Karl Bühlers Sprachtheorie kennen gelernt. Diese Kombination von, sagen wir, Bühler und Hjelmslev, ist nicht ohne innere Schwierigkeiten. Die Glossematik bietet ein sehr abstraktes und eigenwilliges, um nicht zu sagen spekulatives Bild von Sprache. Bühlers 
Karsten Hvidtfelt Nielsen, Braucht eine Textlinguistik Kategorien des Sinns?

Sprachtheorie dagegen trägt die Spuren eines Eklektizismus, den Bühlers umfangsreiche Auseinandersetzungen mit den Kollegen (und Vorgängern) seines Faches sowie auch seine praktischen Erfahrungen als Psychologe und Arzt geprägt haben.

Bei Coseriu führt diese Verbindung von theoretischer Abstraktion und empirischem Detailwissen meistens zu sehr fruchtbaren Synthesen. In einigen Fällen aber scheint Coseriu über die begriffliche Unterschiedlichkeit seiner Voraussetzungen hinweggesehen zu haben; so zum Beispiel in der Einschätzung dessen, was man unter Funktion zu verstehen hat.

In Hjelmslevs Glossematik soll mit Funktion eine abstrakte Abhängigkeit zwischen zwei Größen angegeben werden. In formallogischer Sprache wäre eine glossematische Funktion als zweistellige Relation zu bestimmen. Ob man aber mit solcher Präzisierung die Intention Hjelmslevs genau trifft, ist unsicher ${ }^{45}$. Wenn Bühler von Funktionen spricht, ist eher von dynamischen und wirkenden Prozessen die Rede. Hat ein Sprachelement oder eine Sprachdimension bei Bühler eine Funktion, dann übt das betreffende Phänomen eine konkrete Rolle aus. Die drei berühmten Grundfunktionen seiner Sprachtheorie (Darstellungsfunktion, Appellfunktion, Ausdrucksfunktion) z. B. sind als Angaben von bestimmten Zwecken oder Aufgaben gemeint, die der Sprachverwender mit seinen Sprachhandlungen ausübt, nicht als innersprachliche Abhängigkeiten zwischen abstrakten Größen. Da Coseriu mit seinem Begriff von Funktion eine Verbindung von abstrakten und wirkenden Elementen anstrebt, ist es nicht zu verwundern, dass dieser Begriff bei ihm oft sehr unterschiedliche Aufgaben übernehmen muss.

Die Wirkung von Coserius Entwurf einer Textlinguistik als einer Linguistik des Sinns ist relativ begrenzt gewesen. Das Ausbleiben eines größeren Echos ist wohl vor allem auf die Tatsache zurückzuführen, dass sein Buch gleichzeitig mit der so genannten pragmatischen Wende in der Textlinguistik erschien ${ }^{46}$. Die systemlinguistische Grundausrichtung seines Buches sprach die Befürworter der neuen Textpragmatik wenig an. Ob diese Situation sich mit der gegenwärtigen Zuwendung zu den spezifischen Problemen des Textverstehens ändern wird, bleibt abzuwarten ${ }^{47}$.

Eine Seite von Coserius Textlinguistik müsste eigentlich die Erforscher des Textverstehens interessieren. Mit Nachdruck betont Coseriu, dass er seine Textlinguistik als eine linguistische Hermeneutik verstanden wissen will. Die bei ihm gesuchte Textlinguistik sei, wie er sagt, eine Linguistik, „die für mich mit der richtig verstandenen Philologie und auch mit der Hermeneutik zusammenfällt; die Textlinguistik, in dem Verständnis, die ich Ihnen hier nahebringen möchte, ist nämlich nichts anderes als Hermeneutik," (TL, 35 (meine Hervorhebung)).

Dass Coseriu auch ein ausgewiesener Kenner der Geschichte der Sprachtheorie des Abendlandes war, muss sicher nicht extra gesagt werden ${ }^{48}$. Er hat sich meines Wissens zwar nie direkt mit dem geschichtlichen Fundament der Hermeneutik auseinander gesetzt, weiß (oder wusste) aber selbstverständlich von der Geschichte des europäischen Sprachdenkens genug, um mit der Hermeneutik als Lehre vom Verstehen und vom Schriftsinn gut vertraut zu sein. Die erstere Dimension beherrscht die ganze Anlage der 
Karsten Hvidtfelt Nielsen, Braucht eine Textlinguistik Kategorien des Sinns?

Textlinguistik, die den deutenden Zugang zu sprachlichen Phänomenen konsequent durchhält. Die andere ist sogar in die Definition seines Forschungsprojekts eingegangen. Wie schon angeführt: Diejenige Textlinguistik, der Coseriu mit seiner Einführung das Wort reden will, wird als eine Linguistik des Sinns bestimmt.

Coseriu kommt auf zwei Wegen zu seiner Definition. Der erstere führt über eine Dreiteilung der Arbeitsaufgaben der Linguistik: Im Bereich des Sprachlichen lassen sich, so Coseriu, drei deskriptive Ebenen ausmachen: (a) eine, auf der Sprache als ein universelles Phänomen den zu erforschenden Gegenstand darstellt; (b) eine, auf der die jeweiligen Einzelsprachen studiert werden sollen; (c) eine, auf der, wie Coseriu zunächst sagt, die „Redeakte bzw. [die] Gefüge von Redeakten, die von einem bestimmten Sprecher in einer bestimmten Situation realisiert werden, was natürlich in mündlicher oder in schriftlicher Form geschehen kann" (TL, 7), das Objekt der Sprachbetrachtung bilden sollen.

Was mit den beiden ersten Bestimmungen gemeint ist, leuchtet sofort ein. Mit der dritten aber ist nicht, wie es vielleicht zunächst den Anschein hat, die konkrete Sprachund Textverwendung gemeint. Diese Interpretation wird eigens in einem längeren Exkurs zugunsten einer Bestimmung abgelehnt, die ihre strukturalistische Herkunft nicht verleugnet. Woran Coseriu denkt, lässt sich am besten mit den Vorgaben der strukturalistischen Erzähltheorie vergleichen ${ }^{49}$. So wie das Erzählen den literarischen Strukturalisten als ein Strukturgebilde mit unterschiedlichen Spezialgrammatiken (eine für das Märchen, eine zweite für den Krimi usw.) erschien, will auch Coseriu alle sprachübergreifenden Textformen als besondere Forschungsaufgaben abgesondert wissen. Solche Formen konstituieren die dritte Ebene der Linguistik, auf der nach Coseriu sich z. B. die literarischen Gattungen realisieren (TL, 25-26).

Coseriu erklärt über ein konkretes Beispiel, was er meint: in Kafkas Erzählung Der Bau wird solchen sprachlichen Phänomenen der deutschen Sprache, die auf der zweiten Ebene als konzessive Konjunktionen und Partikeln zu verzeichnen wären, eine zusätzliche Funktion verliehen, mit der die erwähnte dritte Ebene betreten wird. Auf der zweiten Ebene der Sprachbetrachtung lassen sich in Kafkas Text die Vorkommnisse bestimmter Konjunktionen und Partikeln als grammatische Phänomene der deutschen Sprache beschreiben, auf der dritten Ebene aber werden diese Phänomene „zum Zeichen für die Textfunktion Unsicherheit“ (TL, 30). Aus diesem Beispiel generalisiert Coseriu eine Unterscheidung, die für die ganze Anlage seiner Textlinguistik entscheidend wird. Die sprachlichen Zeichen können - mindestens (siehe unten) - zwei Typen von Funktionen ausüben ${ }^{50}$ : entweder als Zeichen einer Einzelsprache oder als Zeichen, die nur auf der Ebene der Texte zum Tragen kommen. Die Ersteren werden Sprachfunktionen, die anderen Textfunktionen genannt. Die Textlinguistik ist nun, nach Coseriu, als eine Hermeneutik zu bestimmen, deren Blick auf Texte über Sprachfunktionen hinwegsieht, um bei der Deutung und der Auswertung von Textfunktionen ihre eigentliche Aufgabe konkretisiert zu finden.

Mit welchem Begriff von Funktion soll nun die Textlinguistik definiert werden? Sollen 
Karsten Hvidtfelt Nielsen, Braucht eine Textlinguistik Kategorien des Sinns?

wir uns unter Funktion eine abstrakte glossematische Abhängigkeit vorstellen, oder soll mit diesem Terminus eher auf Zweck und Wirkung der sprachlichen Phänomene hingewiesen werden? Wie oben angedeutet gibt es bei Coseriu keine klare Antwort auf diese Frage. Überwiegt in den konkreten Analysen die Bühlersche Auffassung von Funktionalität, wird umgekehrt bei der theoretischen Untermauerung des Begriffs explizite auf Hjelmslevs Glossematik hingewiesen.

Mit der Letzteren betreten wir den zweiten Weg, der zu der Definition der Textlinguistik als einer Linguistik des Sinns führt, nämlich die genauere Bestimmung vom Sinn. Für Coseriu scheint nicht Sinn, sondern Inhalt den Oberbegriff für all das, was nicht der phonetisch-graphematischen Seite der Sprache zugeschlagen werden kann, zu bilden. Über die etwaige Ontologie dieses Inhalts erfahren Coserius Leser wenig, wie aber die folgende Diskussion nahe legen wird, scheint Coseriu sich durch die Anbindung an Hjelmslevs abstrakt-formale Zeichendefinition störender Fragen nach dem Wo dieses Inhalts enthoben gefühlt zu haben ${ }^{51}$.

Aus unserer Perspektive lässt sich Coserius Lehre vom Inhalt als eine Lehre von 3fachem Sprachsinn thematisieren. Dass Coserius Lehre sich unter den dreizähligen (wie die von Origines oder Hugo von Sankt Viktor) einreiht, soll selbstverständlich keine geschichtlichen Assoziationen wecken, sondern hat rein systematische Gründe. Die Dreizahl erklärt sich aus Coserius Wunsch, die Lehre vom Inhalt mit der von den drei Dimensionen des Sprachlichen zu korrelieren.

Coserius Darstellung ist an dieser Stelle nicht leicht zu lesen. Man müsste sie eigentlich nach altem hermeneutischem Gebrauch Satz für Satz kommentieren. Das soll aber hier, schon aus Platzgründen, nicht versucht werden. Es gibt drei Klassen von Funktionen: (a) „Die Gesamtheit der Funktionen, die die Bezeichnung von Gegenständen und Sachverhalten in der ,Welt' betreffen“ (TL, 47). Diese werden auf der universellen Ebene der Sprache aktiviert. (b) „Die Gesamtheit dessen, was eine bestimmte Sprache als solche ausdrückt“ (TL, 47). (c) „Und die Gesamtheit der Textfunktionen schließlich, die Gesamtheit dessen, was gerade durch den Text und nur durch den Text verstanden wird“ (TL, 47). Funktionen, die auf der universellen Ebene relevant sind, nennt Coseriu Bezeichnungsfunktionen, Funktionen auf der Ebene der Einzelsprache einzelsprachliche Funktionen, Funktionen auf der Ebene des Textes Textfunktionen ${ }^{52}$. Damit ist die Grundlage für die Lehre vom dreifachen Sprachsinn, vom dreifachen Inhalt, gelegt. Mit Hilfe von Bezeichnungsfunktionen wird eine Art des Inhalts, die Coseriu Bezeichnung nennt, hervorgebracht, über einzelsprachliche Funktionen eine Art, die Bedeutung heißen soll, und mit Textfunktionen eben eine Art, die Coseriu Sinn genannt wissen will ${ }^{53}$.

Die drei Arten des Inhalts hängen nach Coseriu auf eine Weise zusammen, für die er sich die Inspiration in Hjelmslevs Lehre von Metasprachen und Konnotationssprachen geholt hat ${ }^{54}$. Da bei Coseriu (und Hjelmslev) die Kategorien der Sprache sich als formal und relational geben, hat der Linguist das Recht rein kombinatorisch mit ihnen umzugehen. Zum Beispiel steht der Idee nichts entgegen, das, was sich unter einer Betrachtung als Ausdruck (Signifikant) präsentiert, in anderer Verbindung als Inhalt 
Karsten Hvidtfelt Nielsen, Braucht eine Textlinguistik Kategorien des Sinns?

(Signifikat) anzusprechen - und natürlich vice versa. Daher hat Coseriu kein Bedenken, in einem letzten Schritt uns seine Kategorie des Sinns als Signifikat für einen Signifikanten zu präsentieren, der seinerseits aus den beiden anderen Arten des Inhalts, nämlich aus den beiden Signifikaten Bezeichnung und Bedeutung besteht. Also sollen wir uns Texte als Zeichen vorstellen, deren Signifikanten aus den Signifikaten der Sprachzeichen der universellen und der einzelsprachlichen Ebene bestehen.

Nachdem Sinn als die definitorische Kategorie der Textlinguistik auf diese Weise formal festgelegt worden ist, geht Coseriu dazu über sich mit den praktischen Konsequenzen seiner Definition zu beschäftigen. Dies lässt seinen zweiten Gewährsmann zu Worte kommen. In einer längeren Diskussion argumentiert Coseriu dafür, dass Roman Jakobsons bekanntes Modell von Sprache nicht, wie üblich angenommen, einen Fortschritt im Verhältnis zu dem von Karl Bühler darstellt. Das soll nicht heißen, dass Coseriu bereit ist Bühlers Modell ohne weiteres zu übernehmen, nur dass er mit Jakobsons Vorschlägen zur Weiterentwicklung von Bühlers so genanntem Organon-Modell nicht einverstanden ist. Was Coseriu bei Bühler vermisst, ist genau das, was er mit Hjelmslev hat herausbringen wollen: eine formale Bestimmung von Sinn.

In Bühlers Sprachtheorie wird dasjenige, was das menschliche Sprachverhalten von dem der Tiere trennt, eben Sinn genannt ${ }^{55}$. Schon der Verweis auf Sprachverhalten statt auf Sprachsysteme zeigt, dass Bühler mit Sinn kaum dasselbe hat meinen können wie Coseriu. Bei Bühler entsteht Sinn als Resultat realer Prozesse oder, wie er auch sagt, von Funktionen. Dies gibt Coseriu gern für die beiden ersten Bühlerschen Funktionen, Appell und Ausdruck, zu. Bei der Diskussion um Bühlers letzte Funktion, die Darstellungsfunktion, aber wirft Coseriu Bühler vor, dieser habe den Unterschied zwischen dem Vorkommen eines Zeichens in dem Redeakt und dessen Rolle als virtuellem Zeichen übersehen. Hätte Bühler sich diesen Unterschied zwischen konkretem Gebrauch und abstrakter Virtualität klar gemacht, hätte er sein Modell, so Coseriu, um die Kategorie dessen, was bei Coseriu Bedeutung heißt, erweitert (TL, 67).

Es soll hier nicht die Frage aufgeworfen werden, ob mit diesem Vorwurf Bühler Gerechtigkeit widerfährt. Motiviert aber ist der Vorwurf natürlich durch die strukturalistische Vorstellung von dem Zeichencharakter des Sinns. Sinn - an dieser Stelle von Coseriu Bedeutung genannt - vermag die Hjelmslevianische Seite Coserius sich nicht unter den eher prozeduralen Gesichtspunkten eines Bühler vorzustellen. Nach Coseriu kommt Sinn in allen seinen drei Kategorien als eine dem Sprachzeichen immanente Größe vor. Funktionen Hjelmslevianischer oder Bühlerscher Prägung mögen nach Coseriu Sinn vermitteln, bedingen oder bewirken. Sinn selber, sit venia verbo, wohnt als abstrakte Subkategorie des ebenfalls abstrakten Zeichens diesem eigens inne.

Es bleibt abzuwarten, ob das oben angesprochene Interesse für die spezifischen Prozesse des Textverstehens aus Coserius Mischung von Glossematik, Sprachpsychologie und Hermeneutik Inspiration für die eigene Arbeit wird schöpfen können. Dem MDTheoretiker aber hat Coseriu wenig zu bieten. Kein Modell kommt ohne Abstraktionen 
Karsten Hvidtfelt Nielsen, Braucht eine Textlinguistik Kategorien des Sinns?

aus. In einem MD-Modell sind Abstraktionen Idealisierungen, die das Modell nötig hat, um eine sonst unüberschaubare Fülle von konkreten (materiellen) Prozessen und Vorkommnissen überhaupt operationalisierbar zu machen ${ }^{56}$. Bei Coseriu aber (so wie bei seinen strukturalistischen Fachkollegen) wird mit Abstraktionen in einer Art umgegangen, als wäre der Gegenstandsbereich der Linguistik selbst als ein schon an sich abstraktes Feld von Relationen (Funktionen) gegeben ${ }^{57}$. Bei dem Studium dessen, was die Strukturalisten Signifikanten nennen, muss dieser Unterschied zwischen modellhafter und gegenstandsbezogener Abstraktheit keine große Rolle spielen. Es ist für die Forschung relativ gleichgültig, wie die messbaren Daten eingestuft werden, solange es sie nur gibt. Anders aber steht es mit der Erforschung dessen, was hier als Sinn thematisiert worden ist. Ich möchte ungern behaupten, dass es sinnhaften Phänomenen an jeder Art von Existenz fehlt. Das wird ja auch keineswegs von der hier vertretenen Position des epistemischen Monismus verlangt. Wohl aber behaupte ich, dass es bisher keiner Forschung gelungen ist, diese wohl vornehmlich erlebte Existenz an Hand von Messungen oder Beobachtungen wissenschaftlich überprüfbar zu machen. Bei einer solchen Forschungslage wird die Frage nach dem Wo der Abstraktionen brisant (siehe Anmerkung 51). In einem MD-Modell dürfen nur solche Abstraktionen vorkommen, denen materielle Vorkommnisse und deren Träger im Gegenstandsbereich entsprechen. Strukturalisten haben natürlich solche Bedenken nicht. Ihnen genügt der Hinweis auf den abstrakten Charakter ihres Gegenstandbereichs als Rechtfertigung von dessen Existenz.

Anders als Saussure und Hjelmslev scheint Coseriu ein Bewusstsein von den Schwierigkeiten, die mit jeder Sinnforschung verbunden sind, gehabt zu haben. Coseriu gibt unverhohlen zu, dass der Zugang zu den Kategorien des Sinns (des Inhalts) sich nur demjenigen erschließt, der das, was er untersuchen will, schon im Vornhinein intuitiv beherrscht. Die linguistische Beschäftigung mit Textsinn ist für Coseriu deswegen eine hermeneutische oder interpretatorische Aufgabe (TL, 35, 49), weil sich die Leistungen der Textfunktionen erst demjenigen erschließen, der die Sprachfunktionen schon beherrscht. Ob Coseriu sein hermeneutisches Prinzip für alle seine drei Linguistiken reklamieren will, ist mir nicht ganz klar. Sein praktischer Gewährsmann Bühler aber hat dies Prinzip am Anfang seiner Sprachtheorie klar ausgesprochen: „Wie immer man die Sache auch drehen und wenden mag, so muss der sprachforschende Beobachter ganz anders wie der Physiker das mit Ohren und Augen erfasste ... verstehen." (Bühler 1934[65], 12 (Bühlers Hervorhebung)).

Wie dem auch bei Coserius universeller oder einzelsprachlicher Linguistik sei: Die Aufgabe des Textlinguisten ist nach Coseriu eine hermeneutische. MitScherners Worten ließe diese sich als das Überführen von intuitivem Wissen in reflexives Wissen beschreiben (Scherner 1984, 18). Nicht nur Coseriu, sondern die meisten Textlinguisten würden vermutlich dieser Formulierung als einer brauchbaren Umschreibung ihrer Arbeitsbedingungen zustimmen können ${ }^{58}$. Aus der Perspektive, die ich mit diesem Aufsatz vertrete, muss aber selbst reflexives Wissen sich erst über entscheidbare Experimente mit einer materiellen Textverwendungswirklichkeit bewähren, bevor es darauf Anspruch erheben kann als 
Karsten Hvidtfelt Nielsen, Braucht eine Textlinguistik Kategorien des Sinns?

gesichertes Wissen zu gelten. Von einer Textlinguistik, die sich solchen Kriterien stellen kann, haben wir uns mit dem Übergang von Frege zu Coseriu eher entfernt. Versuchen wir deshalb uns mit dem letzten Modell unserem Ziel erneut zu nähern.

\subsection{Luhmann}

In Coserius Textlinguistik ließen sich zwei innere Spannungen ausmachen: eine, die den Funktionsbegriff zwischen zwei unterschiedlichen Modellierungen schwanken lässt, und eine, die sich aus dem bald formalen, bald hermeneutischen Zugang zu dem Studium von Sinn nährt. Wollen wir die erstere auf die Personen Hjelmslev und Bühler verteilen, können wir die letztere als ein Hin und Her zwischen glossematischen und hermeneutischen Inspirationen interpretieren.

Bei Luhmann, unserem letzten Sinntheoretiker, treten den Textlinguisten ganz andere Gegensätzlichkeiten entgegen. Es soll hier weder versucht werden, einen Überblick über Luhmanns Bedeutung für die Entwicklung soziologischer Modelle zu geben, noch die wissenschaftstheoretische Debatte, zu der Luhmanns Konstruktivismus Anlass gegeben hat, nur annäherungsweise zu skizzieren. In den Gesellschafts- und Kulturwissenschaften wird Luhmann zur Zeit als konstruktivistische Variante von oder Pendant zu Foucaults diskurstheoretischem Ansatz diskutiert ${ }^{59}$. Ob sich die Textlinguistik in einer oder mehreren von ihren Varianten von Luhmann hat inspirieren lassen, ist mir, bei aller Plausibilität der Hypothese, nicht bekannt ${ }^{60}$.

Luhmanns Oeuvre ist von einschüchternden Dimensionen, sowohl im Umfang als im Wissen. Da es mir in diesem Kontext darum geht, Luhmann als Sinntheoretiker darzustellen, werde ich mich auf die für diesen Aspekt relevanten Seiten seiner Gesellschaftstheorie beschränken. Aus Übersichtlichkeitsgründen halte ich mich hauptsächlich an die große Gesamtbilanz der Theorie, wie Luhmann sie mit dem zweibändigen Werk Die Gesellschaft der Gesellschaft (1998) vorgelegt hat ${ }^{61}$.

In Luhmanns Gesellschaftstheorie ist Phänomenen wie Kommunikation, Sinn und Sprache großer Platz eingeräumt. Obwohl Luhmanns Spezialwissen auf dem Bereich der Linguistik wohl eher begrenzt war, lässt sich doch in seiner sprachbezogenen Begrifflichkeit eine gewisse Vorliebe für strukturalistische und, insofern man solche der Linguistik zuschlagen will, poststrukturalistische Positionen spüren. Selbst verweist Luhmann auf Theoretiker wie Saussure (GG, 195, 208), Barthes (GG, 208), Derrida (GG, 75) und de Man (GG, 33, 95) ${ }^{62}$. Aber vielleicht hätte eine Berufung auf die strukturalistische Sonderform der Glossematik Luhmanns Anliegen noch besser geziemt. Wie Hjelmslev geht auch Luhmann von einer strikten Teilung von Form und Substanz aus. Für beide kommt nur die Form als Forschungsobjekt in Frage. Unter Form wird bei beiden eine Relation oder eine Differenziation verstanden ${ }^{63}$, und beide sind gleich bereit sämtliche Konsequenzen, die sich aus diesen Prinzipien ergeben, mit größter Radikalität zu befolgen.

Aus textlinguistischer Warte lässt sich zwischen Hjelmslev und Luhmann eigentlich nur 
Karsten Hvidtfelt Nielsen, Braucht eine Textlinguistik Kategorien des Sinns?

ein einzelner wesentlicher Unterschied ausmachen. Hjelmslevs Glossematik stellt sich das System der Sprache als ein starres Gefüge von Differenzen vor, die alle in abstrakter Gleichzeitigkeit dem Forscher zugänglich sind. Auch aus Luhmanns Perspektive ließen sich Sprachen als relationale Systeme charakterisieren, doch deren Relationsgeflecht soll in seiner ständig sich wandelnden Zeitlichkeit begriffen werden. Wo Hjelmslev unter einer These von Synchronizität sich Sprache als eine formal-abstrakte Festigkeit zurechtlegt, versucht Luhmann Form und Zeit auf eine höchst originelle (und nicht immer leicht verständliche) Weise zu verbinden. Wenn man will, kann man diesen theoretischen Unterschied beim gemeinsamen Formdenken mit dem verbalen von Differenz und Differenzierung verdeutlichen (oder dem von Unterschied und Unterscheidung). Hjelmslev sind Systeme Gefüge von stabilen Differenzen, Luhmann fasst jene als dynamische Strukturen von zeitgebundenen Differenzierungen auf.

Zur Temporalisierung kommen Systeme nach Luhmann mit Hilfe von Rekursion. Es ist schwer den Konsequenzen dieses Rekursionsbegriffes bei Luhmann in allen Einzelheiten intuitiv zu folgen, geschweige denn theoretisch gerecht zu werden. Unten soll versucht werden anzudeuten, wie ein Modell von Sprachsinn auszusehen hätte, das Luhmanns Forderungen genügen würde. Wir werden sehen, dass Luhmanns Mischung von, sagen wir, Glossematik und Rekursion uns einen völlig anderen Blick auf Sinn gestatten wird als Coserius Bemühung um eine hermeneutisch angereicherte Glossematik.

Wenn Luhmann von Systemen spricht, wird zunächst von formalen Größen gesprochen. Systeme konstituieren sich, indem sie die Unterscheidung zwischen sich und einer materiellen Umwelt, in der sie wirken, treffen. Auch die materielle Seite der Agenten eines Systems - seien sie Tiere, Menschen oder Automaten - gehört zur Umwelt. In Hjelmslevs Worten: ein System ist eine Form, seine Umwelt deren Substan $z^{64}$.

Luhmanns Interesse gilt in erster Linie sozialen Systemen. Die sind vor allem dadurch charakterisiert, dass sie das Phänomen Kommunikation produzieren und reproduzieren. Um genauer zu beschreiben was wir uns an dieser Stelle unter Kommunikation vorstellen dürfen, muss vorerst der Begriff des Mediums abgeklärt werden. „Kommunikationssysteme konstituieren sich selbst mit Hilfe einer Unterscheidung von Medium und Form." (GG, 195). Diese Unterscheidung ist, mit Luhmanns Worten, „stets ein systeminterner Sachverhalt." (GG, 195). Als Differenzierung ist diese Unterscheidung von Medium und Form selbst eine Leistung des Systems und also keine, die dem System von außen aufgezwungen worden wäre. Nach Luhmann funktioniert ein System in einem Modus, der über andauernde Ausdifferenzierungen zu immer neuen Verzweigungen im System führt. Aus jeder Ausdifferenzierung gehen neue Ausdifferenzierungen hervor. Somit besagen die beiden angeführten Zitate, dass ein generelles System zu einem Kommunikationssystem ab dem Augenblick mutiert ist, nach dem es im System die Differenzierung von Form ${ }^{65}$ und Medium gibt.

Die Differenzierung von Form und Medium vergleicht Luhmann mit der bei Saussure von langue und parole. Das soll vielleicht nicht ganz buchstäblich genommen werden. Jedenfalls ist es schwierig zu verstehen, wie die Differenzierung zwischen Form und 
Karsten Hvidtfelt Nielsen, Braucht eine Textlinguistik Kategorien des Sinns?

Medium „stets ein systeminterner Sachverhalt" bleiben kann, wenn Medium dasselbe wie parole bezeichnen sollte ${ }^{66}$. Aus Luhmanns Sicht muss mit Medium ein systeminternes, also langue-spezifisches Element gemeint sein. Also ist auch Medium eine Form (ein System besteht nur aus Formen, Unterscheidungen). Für diese Form gibt es - ganz in Hjelmslevs Geiste - auch bei Luhmann eine Substanz, die meistens mediales Substrat genannt wird. Anders als das Medium gehört das mediale Substrat zur Umwelt und hat als solches mit dem System nichts zu tun.

Die Trennung von Medium und Form lassen Systeme kommunizieren. Zwei Typen von Systemen kommunizieren in dem besonderen Medium, das Luhmann Sinn nennt: soziale und psychische Systeme. Für Luhmann ist Sinn deshalb ein Grundbegriff der Soziologie so wie der Psychologie. Unten werden wir uns zu fragen haben, ob ein Ähnliches für eine entsprechend modifizierte Textlinguistik auch zu gelten hat.

Soziale Systeme sind aus Formen, aus Unterscheidungen konstruiert. Diese sind aber nicht, wie im Strukturalismus und in der Glossematik, als starre Relationsgefüge zu verstehen, sondern, wie gesagt, von einem dynamisierenden Phänomen geprägt, das Luhmann Rekursion nennt. Damit will Luhmann auf das gleichnamige mathematische Phänomen verweisen, ohne sich jedoch zu dessen technischer Formalität verpflichtet zu fühlen ${ }^{67}$. Es soll auch hier nicht versucht werden, Luhmanns Zentralbegriff auf dessen mathematische Voraussetzungen zurückzuführen, doch werde ich - wie bei der Präsentation meines MD-Modells - mich eines bescheidenen Grads von Formalisierung bedienen, um die Implikationen dieses vielschichtigen Begriffs bei Luhmann einigermaßen festhalten zu können.

In grober Vereinfachung kann man das Wirken einer rekursiven Funktion mit folgender Symbolik wiedergeben:

1) $\quad \mathrm{f}_{\mathrm{n}}\left(\mathrm{f}_{\mathrm{n}-1}\left(\mathrm{f}_{\mathrm{n}-2}(\ldots(\Delta) \ldots)\right)\right)$

Intuitiv ist (1) so zu lesen, dass der Wert einer jeglichen ,inneren' Funktion das Argument der sie unmittelbar umklammernden abgibt. Mathematiker brauchen eine konstante Funktion, um den Prozess der Rekursion zu starten. Die sei hier mit $\Delta$ angedeutet, soll aber im Folgenden ausgespart bleiben ${ }^{68}$. In der Mathematik spielt die zeitliche Dimension der Rekursion keine besondere Rolle. Das ist bei Luhmann ganz anders. Wie unten ausführlicher zu besprechen sein wird, spielt der Zeitfaktor eine absolut entscheidende Rolle im begrifflichen Apparat, der Luhmanns Systemdenken trägt. Um dieser Rolle zunächst formal gerecht zu werden, legen wir fest, dass die Indizierung der Funktionen in (1) stets auch als Zeitangabe gelesen werden soll: $f_{n-2} z$. B. liegt zeitlich unmittelbar vor $f_{n-1}$, und genereller gesprochen: Seien $f_{i}$ und $f_{j}$ zwei rekursive Funktionen in derselben Rekursionsreihe, dann gilt, dass $\mathrm{f}_{\mathrm{i}}<_{\mathrm{t}} \mathrm{f}_{\mathrm{j}}$ genau dann, wenn $\mathrm{i}<_{\mathrm{a}} \mathrm{j}$ (wobei $<_{\mathrm{t}}$ eine temporale Vor-nach Relation ist, $<_{\mathrm{a}}$ die ,normale' arithmetische Relation für natürliche Zahlen).

Unter Sinn sollen wir uns nun eine Form (Differenzierung) vorstellen, deren weitere Differenzierungen immer als Ergebnisse von temporalisierten Rekursionen vorkommen ${ }^{69}$. Diese Bestimmung ist keineswegs erschöpfend. Unten sollen einige zusätzliche 
Karsten Hvidtfelt Nielsen, Braucht eine Textlinguistik Kategorien des Sinns?

Bedingungen, die das Phänomen Sinn bei Luhmann zu erfüllen hat, angeführt werden. Da aber Sinn grundsätzlich rekursiver Natur ist, soll zunächst versucht werden unter Bezug auf (1) dessen nicht leicht zu verstehende Existenzweise zu veranschaulichen.

Wie bei dem MD-Modell sehen wir von Evolutionsfragen ab. Daher werden wir uns wenig darum kümmern, wie es im Verlaufe der Evolutionsgeschichte sozialer Systeme zu einem Phänomen wie Sinn überhaupt gekommen ist ${ }^{70}$. Wir betrachten das System zu einem Zeitpunkt, wo es schon Sinn gibt. Wir gehen weiter davon aus, dass Sinn rekursiv kommuniziert wird. Diese Operation wollen wir nun mit Hilfe von (1) näher bestimmen. Unter unserer temporalisierten Perspektive lässt die rekursive Funktion $\mathrm{f}$ sich als eine Reihe von Einzelfunktionen $\mathrm{f}_{\mathrm{t}-1}, \mathrm{f}_{\mathrm{t}}, \mathrm{f}_{\mathrm{t}+1}$ etc. verstehen. Jede Einzelfunktion nimmt als Argument den Wert der unmittelbar vorhergehenden. Wie dürfen wir uns solche Argumente und Werte vorstellen? Wäre die Sache rein mathematisch abzuhandeln, hätten wir zwei Möglichkeiten: Wir könnten Argumente und Werte entweder als Zahlen oder als Funktionen definieren. Wählten wir die erstere Möglichkeit, hätten wir uns für eine doppelte (normale mathematische) ,Ontologie' entschieden, denn außer Einzelfunktionen gäbe es dann in unserer mathematischen Welt auch feste numerische Größen. Mit der zweiten Möglichkeit bestückten wir unsere Welt ausschließlich mit Funktionen: Jede Einzelfunktion nähme dann als Argument ihre unmittelbare Vorgängerin und produzierte als Wert diejenige Funktion, die ihrer unmittelbaren Nachfolgerin als Argument diente. Welche ,Lesart' sollen wir wählen?

Die rein mathematische Sichtweise stellt ohne Zweifel sowohl eine technische Überforderung als auch eine theoretische Vereinfachung von Luhmanns Sinnbegriff dar. Bei dem Versuch mit dessen methodischen Implikationen ins Reine zu kommen, mag der mathematische Blick aber hilfreich sein. Was Luhmann mit seiner Betonung der rekursiven Natur von Sinn herausbringen will, lässt sich, glaube ich, mit der zweiten Möglichkeit am ehesten darstellen. Also beschränken wir uns auf eine mathematische Welt, die nur Funktionen kennt. Danach wird man sich Sinn zunächst als Folgen von temporalisierten Funktionen vorstellen dürfen, deren Rekursivität durch (2) angedeutet werden kann:

2) $\quad \mathrm{f}_{\mathrm{t}}\left(\mathrm{f}_{\mathrm{t}-1}\right)=\mathrm{f}_{\mathrm{t}+1}{ }^{71}$

Die Funktion $f_{t}$ wirkt auf $f_{t-1} u m f_{t+1}$ hervorzubringen. Dasselbe Muster wird von jedem Tripel $\left\langle\mathrm{f}_{\mathrm{t}-1}, \mathrm{f}_{\mathrm{t}^{\prime}}, \mathrm{f}_{\mathrm{t}+1}>\right.$ von Funktionen durchgespielt. Es gibt keine Stelle, wo eine Größe, die nicht selbst Funktion wäre, als Mittelglied auszusondern wäre. Restlos wird Sinn als Funktion von Funktionen kommuniziert. Dazwischen liegt nichts, was sich als selbständige Argumente oder Werte bestimmen ließe. In einer ,realistischeren' Modellierung davon, wie Sinn in und von Systemen kommuniziert wird, käme man natürlich nicht mit nur einer Funktionsfolge aus, sondern müsste ein Gefüge von Funktionsfolgen angeben, die auf eine Art, die schnell jeder Modellierung spotten würde, ineinander zu verflechten wären.

Sich Sinn als temporalisierte Folgen von rekursiven Funktionen zurechtzulegen, 
mag hilfreich sein, zwei Leitgesichtspunkte zu erklären, die in Luhmanns Theorie von erheblicher Wichtigkeit sind. Auf den ersten ist schon hingewiesen worden: Sinn ist ein Medium, das heißt eine Form, was wiederum bei Luhmann dasselbe wie eine Unterscheidung heißen soll. Es gibt nur Sinn als den andauernden Prozess der temporalisierten Ausdifferenzierung, nie als verfügbare „zeitüberdauernde[n] Identitäten“ (GG, 45). Sinn ist kein „Vorhandenes“ (GG, 45), sondern ein, sagen wir, Prozessuales. Fragt man nun, wie - wenn überhaupt - man sich diese Prozessualität in ihren konkreten Auswirkungen vorstellen darf, wird deutlich, dass Luhmann sich Sinn weit weniger abstrakt als z. B. Hjelmslev vorgestellt hat. Anders als bei den Strukturalisten hat Sinn eine durchaus, konkrete' Erscheinungsform, die nicht aus dem System der Sprache in deren parole zu verbannen wäre. Wie wir bald sehen werden, ist diese ,Konkretheit' nur die eine Seite von einer Bestimmung, die man am Ende weder konkret noch abstrakt nennen möchte (daher die Anführungszeichen). Aber bei aller gebotenen Vorsicht geht man wohl an Luhmanns Intentionen nicht völlig vorbei, wenn man dem Prozessieren von Sinn einen Ereignischarakter zuschreibt. Sinn ist eine Prozessualität, die sich in Ereignissen manifestiert ${ }^{72}$. Auch scheint klar, dass das Wo von diesen Ereignissen als eine „'in den Köpfen von Menschen' individualisierte Realität" anzugeben ist (GS, 18). Dabei soll nicht vergessen werden, dass die jeweiligen Bewusstseine der sinnkommunizierenden Agenten keine Rolle für die systemische Beschreibung von Sinn spielen dürfen. Zwar ereignet sich Sinn in und mit den Agenten eines Kommunikationssystems. Die aber sind für das Wirken des Systems und dessen Sinnereignisse völlig gleichgültig. Dies zu verstehen gehört zu einer der größten Herausforderungen, die der Luhmannsche Sinnbegriff den Textlinguisten stellt.

Der zweite Leitgesichtspunkt lässt sich zunächst rein formal ableiten. Sinn ist ein Medium. Damit ist ein differenzierender Prozess gemeint, zu dessen Funktionsweise es gehört, Unterscheidungen zu aktivieren. Das System lässt Sinn entstehen als die Unterscheidung von Form und Medium. Diese Unterscheidung produziert ihrerseits eine erneute Ausdifferenzierung, die alle Sinnereignisse gleichmäßig charakterisiert, nämlich die von Aktualität und Potenzialität (GG, 50, 58).

Diese Differenzierung kann man, wie mir scheint, auf zweierlei Weise näher bestimmen. Auf einem ,begriffslogischen' Niveau wiederholt die Struktur des Sinns die generelle Struktur einer Form. Die Unterscheidung, die mit (und in der Gestalt) einer Form getroffen wird, wirkt sich als eine Demarkationslinie zwischen zwei Möglichkeiten aus. Die eine Seite wird mit der Aktivierung der Form markiert ${ }^{73}$, die andere bleibt als blinder, aber für die Markierung notwendiger Fleck bei der Unterscheidungsoperation zurück $(G G, 69)$. Die markierte Seite erhält den Status einer aktualisierten Sinnkomponente, die blinde den einer potenziellen.

Der Ereignischarakter von Sinn zeigt sich auf dessen aktualisierter Seite. Da aber diese Seite ohne ihre nicht-aktualisierte, also nur potenzielle Seite nicht gedacht werden darf, erschöpft sich Sinn in dessen konkretem sich Ereignen nicht (daher können jetzt Anführungszeichen um konkret ausgelassen werden). Das konkrete Ereignis, können wir 
Karsten Hvidtfelt Nielsen, Braucht eine Textlinguistik Kategorien des Sinns?

vielleicht sagen, ist immer auch ein nicht-konkretes Nicht-Ereignis. Das Aktualisierte gibt es nur, weil es auch ein Potenzielles gibt, das als Reservoir für zukünftige Aktualisierungen dienen kann. Es soll hier nicht versucht werden, diese Bestimmung auf ihre etwaige textlinguistische Plausibilität hin zu diskutieren ${ }^{74}$. Dass aber Sinn sich in der Doppeltheit von Aktualität und Potenzialität ereignet, mag uns verstehen helfen, wie Luhmann zu der Depersonalisierung seiner Sinntheorie (Systemtheorie) kommt. Das Ereignis von Sinn findet in den Agenten des jeweiligen Systems statt (bei Menschen in deren Bewusstsein). Aus diesem Befund aber schließen zu wollen, die Agenten des Systems ließen sich über das Vorkommnis von Sinn abfragen, ist nach Luhmann ein Missverständnis. Zu Sinn als Systemgröße gehört, wie wir eben erfahren haben, auch das, was den Einzelindividuen eben nicht zugänglich ist. Kommunikation spielt sich in einem Zwischenreich von Wissen und Nichtwissen ab. Und Individuen über ihr Nichtwissen befragen zu wollen, hat nach Luhmann geringe Aussichten. „Es ist ... völlig unrealistisch, anzunehmen, ein Individuum wisse, was es nicht wisse.“ (GG, 39)75. Daher kann Luhmann mit einiger Konsistenz behaupten, „keiner der an der Kommunikation Beteiligten kann wissen, wie das im einzelnen geschieht." (GG, 73). Die Agenten eines Systems nehmen am Wirken des Systems teil, sind aber lediglich als austauschbare Träger eines Geschehens zu verstehen, das jenseits von Bewusstsein und individueller Kontrolle sein rekursives Spiel treibt.

Sinn ist das generelle Medium der Kommunikation. Bis jetzt haben wir dies Medium nur in höchster Formalität kennen gelernt. Aus Sinn geht die Unterscheidung von Aktualität und Potenzialität hervor. Textlinguisten mag diese Bestimmung noch relativ exotisch anmuten. Sie wird aber auf einem zweiten Niveau expliziert, dem seine Verwandtschaft mit herkömmlichen Modellierungen von Kommunikation etwas deutlicher anzumerken ist.

Auf den ersten Blick scheint Luhmann für sein generelles Modell für Kommunikation bei etwa Bühler Anleihe gemacht zu haben. So wie die Kommunikation nach Bühler drei Funktionen umschließen muss, Ausdrucksfunktion, Darstellungsfunktion und Appellfunktion, denkt sich auch Luhmann das gesamte Kommunikationsereignis in drei Phasen aufgeteilt: in Information, Mitteilung und Verstehen. Nun soll diese Phasenlehre mit der formalen Bestimmung von Sinn als die Unterscheidung von Aktualität und Potenzialität verbunden werden. Das geschieht über den Begriff von Selektion. Aus ,begriffslogischer' Warte lässt sich die erforderte Verbindung leicht erstellen. Wir müssen nur den Begriff der Selektion als ein sinntheoretisches Äquivalent für Unterscheidung lesen, damit wir uns die Unterscheidung von Aktualität und Potenzialität als eine Selektion zurechtlegen können, bei der das aktualisierte Moment gleichzeitig das selegierte abgibt. In allen drei Kommunikationsphasen ist also zwischen dem, was in aktualisierter Form, und dem, was unter dem Schleier der Potenzialität herumgereicht werden soll, zu unterscheiden. Was herumgereicht wird, ist natürlich Sinn. Es fragt sich aber, was man sich bei dessen dreiphasiger Selektion in dem Kommunikationsereignis näher vorstellen darf. 
Karsten Hvidtfelt Nielsen, Braucht eine Textlinguistik Kategorien des Sinns?

Zunächst hat es allen Anschein, als führte Luhmanns Modellierung von Kommunikation in Textlinguisten vertrautes Gelände. Als Information wird unter Vorwegnahme verstehensrelatierter Erwartungen aus der Fülle der möglichen Sinnkonstellationen eine überraschende ausgewählt, die dem jeweiligen Interessenstand des Systems entsprechen soll (GG, 71-72). Nach der Selektion von Information folgt die von Mitteilung. An mehreren Stellen (GG, 85, 124, 190, 442) betont Luhmann, dass es ohne die Unterscheidung von Information und Mitteilung gar nicht zur Kommunikation kommen kann, hat aber darüber hinaus wenig über die zweite Kommunikationsphase zu sagen. Was bei der Mitteilung eigentlich selegiert wird, ist (mir) nicht ganz klar (geworden). Doch vielleicht verweist folgende Bemerkung bei Luhmann auf eine mögliche Spezifikation von dem, was die Mitteilungsselektion für Sinn tut: „Jede Kommunikation muss zugleich kommunizieren, dass sie eine Kommunikation ist ... " (GG, 86). In textlinguistischer Sprache könnte man die Illokutionsleistungen einer Aussage als deren Verweis auf den kommunikativen Charakter des Sprachereignisses betrachten. Mit der Wahl einer bestimmten Illokutionsform wird eine - die gewählte - aktualisiert und die zahlreichen anderen in reine Potenzialität zurückversetzt. Dabei wird mit gleichgültig welcher Illokution auch immer mitkommuniziert, dass das Sprachereignis (die Textverwendung) als Kommunikation zu gelten hat. Propositionen könnte man zur Not als generelle ,Inhalte' modellieren, die es (auch) unabhängig von den Kommunikationsakten gibt, Illokutionen wohl kaum. Ob aber Luhmann sich Ähnliches unter seiner Mitteilungsselektion vorgestellt hat, ist mehr als ungewiss.

Die letzte Phase im Kommunikationsereignis wird von einer Komponente, die Luhmann Verstehen nennt, ausgemacht. Nach Luhmann hat der systemtheoretische Kommunikationsforscher „nicht von der Sprachhandlung [...] sondern von der Situation des Mitteilungsempfängers" (GG, 210) auszugehen. Scheinen die Komponenten Information und Mitteilung eher in die Kompetenz eines Senders zu fallen, wird Verstehen bei Luhmann dem Empfänger zugeschlagen. Verbreitungstechnologien wie die der Schrift (und die der IT) haben die frühere Einheit des Kommunikationsereignisses aufgebrochen und dazu geführt, dass dies Ereignis sich „zeitlich und räumlich an viele Adressaten verteilen und damit zu unvorhersehbar vielen Zeitpunkten realisieren kann“ (GG, 71). Aber auch bei größter zeitlicher Distanz kommt das Kommunikationsereignis erst mit dem Verstehen zum Abschluss. Es erhellt aus Luhmanns Modellierung, dass die Kombination von Information und Mitteilung zu Verstehen in keiner Eins-zu-eins Relation stehen kann. Jedem Paar von Information und Mitteilung, das den erwähnten Verbreitungstechnologien überantwortet worden ist, wird eine unbegrenzte Zahl von Verstehensereignissen entsprechen können. Auch Verstehen ist eine Selektionsleistung, die man sich als eine Reihe von Sinnaktualisierungen vorstellen darf, mit der eine Reihe von nicht realisierten Sinnpotenzialitäten parallel läuft.

Wenig in der hier versuchten Präsentation von Luhmanns Kommunikationsmodell müsste herkömmlichen textlinguistischen Vorstellungen widersprechen. Dass aber das Luhmannsche Modell sich unter ganz anderen Prospekten als denjenigen, die nach Bühler entstanden sind, zu konzeptualisieren ist, ist offensichtlich. Bei Luhmann 
Karsten Hvidtfelt Nielsen, Braucht eine Textlinguistik Kategorien des Sinns?

kommunizieren keine Einzelindividuen, sondern das System selbst. Die Selektionen, mit denen das kommunikative Ereignis zu formatieren ist, sind keine voneinander unabhängigen Leistungen, für die die Kommunikationspartner zeichneten. „Die Komponenten der Kommunikation setzen einander wechselseitig voraus; sie sind zirkulär verknüpft. Sie können daher ihre Externalisierungen nicht mehr als Eigenschaften der Welt ontologisch fixieren, sondern müssen sie im Übergang von einer Kommunikation zur anderen jeweils suchen." (GG, 72).

Mit dieser Warnung soll Textlinguisten jeder Sorte ernsthaft ins Gewissen geredet werden, dass sie der Versuchung nicht nachgeben, die eben skizzierte Modellierung eines kommunikativenEreignissesumstandsloseigenenBegriffenanzugleichen.Strukturalisten sollen zu verstehen suchen, dass Luhmanns Modell bei aller kommunikativen Anlage als eine rein systemische Angelegenheit zu betrachten ist. Pragmatiker sollen sich hüten, die eben angedeutete Dreiteilung des Kommunikationsprozesses mit konkreten Kommunikationsakteuren zu bestücken. Und Hermeneutiker (und Kognitivisten) gehen an Luhmanns Intentionen vorbei, wollten sie in Luhmanns Begriff von Verstehen ein Äquivalent eigener Modellierungen finden.

Die Kommunikation ist eine Leistung des Systems. Die Selektion von Information ist keineswegs unter Annahme einer selegierenden Senderintentionalität zu begreifen. Die Wahl der Mitteilungsform steht auch nicht im Befinden der konkreten Teilnehmer an der Kommunikation, sondern wird ,hinter deren Rücken' vom System vorgenommen. Und schließlich ist mit Verstehen kein hermeneutischer (oder kognitiver) Prozess gemeint, der in Einzelindividuen zu orten wäre, sondern eine Schaltstelle des Systems in dessen weiterem Prozessieren von Sinn.

Die Kommunikation besteht aus transienten und rekursiven Ereignissen (GG, 71). Mit diesen Ereignissen wird das Medium Sinn herumgereicht, aber nie auf eine Weise, die es dem Medium gestattet, sich zu zeitüberdauernden semantischen Identitäten zu verfestigen. Daraus sollen keine Fregeschen Konklusionen gezogen werden. Die Kategorie des Sinns ist nicht in die Privatheit nicht-kommunizierender Individuen verbannt. Luhmann ist kein Theoretiker vom 0 -fachen Textsinn. Mit einer paradoxen Formulierung, auf die Luhmann Wert legt, ist Sinn „ein endloser, also unbestimmbarer Verweisungszusammenhang, der in bestimmter Weise zugänglich und reproduziert werden kann." (GG, 49-50) ${ }^{76}$. Wollte man daher Luhmanns Sinnbegriff in die Lehre vom n-fachen Schriftsinn einordnen, wäre $\mathrm{n}$ bei ihm mit einer nach oben unbegrenzten Kardinalität anzugeben.

Sinn ist unentscheidbar ${ }^{77}$, aber diese Unentscheidbarkeit wird durch Rekursion operationalisierbar gemacht. Anders ausgedrückt: wäre Sinn - wie bei Frege die Vorstellungen - radikal unentscheidbar, dann hätte Sinn keine systemische Rolle zu spielen $^{78}$. In Kommunikationssystemen kommt es durchaus zu Kondensierungen von Sinn, die über iterative Anwendung von Sinnverarbeitungsregeln eine gewisse Verfügbarkeit von Sinn zulassen. Solche Kondensierungen hat Luhmann mehrmals unter dem Namen von Semantik studiert ${ }^{79}$. 
Karsten Hvidtfelt Nielsen, Braucht eine Textlinguistik Kategorien des Sinns?

Die Sprache ist für Luhmann das grundlegende Kommunikationsmedium einer Gesellschaft (GG, 205). Die Sprache ist eine Form (GG, 213), die die Unterscheidung zwischen Sinn und Laut trifft. Mit Laut ist selbstverständlich, wie bei Saussure und Hjelmslev, nicht das physische Phänomen, sondern der formale Signifikant gemeint ${ }^{80}$. Daher lässt sich sprachliche Kommunikation als „Prozessieren von Sinn im Medium der Lautlichkeit" (GG, 213) bestimmen"

Bis jetzt ist wenig von demjenigen Aspekt von Luhmanns Theorie, der wohl die meisten seiner Leser fasziniert oder irritiert hat, die Rede gewesen. Luhmann behauptet, dass Sinn, als dem generellen Medium für kommunizierende Systeme wie das soziale und das psychische, eine nicht überschreitbare Geschlossenheit innewohnt. Die Form des Sinns, so Luhmann, „ist das absolute Medium ihrer selbst.“ (GG, 57). Oder anders gewendet: „Das letzte, für Sinnsysteme nicht transzendierbare Medium ist deshalb der Sinn.“ (GG, 59).

Wie in der Transzendentalsemiotik eines Apel ${ }^{82}$ die Sprache, spielt bei Luhmann das Phänomen des Sinns die Rolle eines nicht hintergehbaren Aprioris. Soziale und psychische Systeme operieren und kommunizieren nach Luhmann in diesem nichtüberschreitbaren Medium. Aus dieser These erklären sich die meisten Faszinosa und Irritationsmomente, zu denen Luhmanns Systemtheorie Anlass gegeben hat. Diese sollen hier nicht einzeln aufgezählt werden. Ich werde mich auf solche beschränken, die für die Art, wie ein Modell von Sinn nach Luhmann anzugeben wäre, heranzuziehen sind.

Luhmann will seine Sinntheorie (Systemtheorie) als Teil einer neuen intellektuellen Entwicklung betrachtet wissen, die „die alte Gegenüberstellung von Natur- und Geisteswissenschaften oder hard sciences und humanities oder gesetzesförmig bzw. textförmig (hermeneutisch) gegebenen Gegenstandsbereichen zu unterlaufen“ (GG, 60) ermöglicht. Was damit gemeint werden kann, lässt sich in unserem Zusammenhang über einen Vergleich mit strukturalistischen Modellierungen von Sinn verdeutlichen. Bei Hjelmslev z. B. wäre unter dem glossematischen Terminus Inhaltsform das Phänomen Sinn als ein abstraktes Gefüge von Differenzen zu modellieren. Wie früher angeführt, ginge diese Abstraktheit nicht auf Kosten des Modellbauers, sondern wäre als Eigenschaft des zu modellierenden Gegenstandsbereichs hinzunehmen. Der Sinnforscher steht diesem abstrakten Bereich mit derselben Strenge und Unabhängigkeit gegenüber, wie sie sein naturwissenschaftlicher Kollege in seinem Studium von materiellen Objekten voraussetzt ${ }^{83}$.

Dies ist bei Luhmann anders. Ich habe mehrmals auf eine besondere Schwierigkeit hingewiesen, die sich aus Luhmanns Begriffen ergibt. Diese scheinen sich gewöhnlichen Dichotomien zu widersetzen, wie z. B. der von konkret und abstrakt. Einerseits wären die Sinnereignisse, von denen Luhmann spricht, als konkrete Handlungen zu modellieren, andererseits fehlt ihnen jeder Bezug zu den Agenten, ohne die man normalerweise sinnbezogenen Handlungen keine Konkretheit würde zuschreiben können. Ich habe es absichtlich vermieden, diese Ereignisse als abstrakt zu qualifizieren. Luhmann ist 
kein Hjelmslev, dem eine postulierte Abstraktheit genügt. Auch scheint Luhmann keinen Anschluss bei denen gesucht zu haben, die sich für Sinn eine mentale oder psychische Ontologie ausgedacht haben. Sinn bei Luhmann ist weder als konkretes noch abstraktes, weder als materielles noch mentales Phänomen zu modellieren.

Wollte man ein Wort für das, was Luhmann in seinem Modell von Sinn als dessen Existenzweise unterbringen möchte, müsste man auf eine Tautologie zurückgreifen. Von seiner rekursiven Temporalität abgesehen hat Sinn die fundamentale Eigenschaft sinnhaft zu sein. Damit soll auf die oben angesprochene Universalität von Sinn hingewiesen werden. Sinn ist eine Dimension, die so wenig wie die Zeit, in der sich Sinn bewegt, aufgesprengt werden kann. Von außen betrachtet gibt es Sinn nicht. Sinnfreie Systeme wie die physikalischen (das Wetter z. B.) brauchen keine Bezüge auf Sinn, um funktionieren zu können (um regnen zu können). Sinnkommunizierende Systeme, wie die sozialen oder die psychischen, können gar nicht aus dieser Dimension heraustreten. Auch die Agenten solcher Systeme sind für ihre Wahrnehmung und Beobachtung auf diese Dimension völlig angewiesen. Aus dem Innern eines Kommunikationssystems kann keine Umwelt beobachtet und beschrieben werden, sondern registrieren lässt sich nur, wie diese sich in den schon vom System bereitgehaltenen Kategorien von Sinn darstellt ${ }^{84}$. Kein Agent vermag aus dieser Haft herauszutreten, die niemand gebaut, geschweige denn gewollt hat. Sinn ist sein eigener Urheber, Mittler und Vollstrecker. Er ist in keinem dritten Reich wie bei Frege zu lokalisieren, sondern als diejenige Innenseite, mit der soziale und psychische Systeme ihre Agenten lückenlos umschließen.

Von solcher Einsicht aus ist bei Luhmann die Trennung von Subjekt (Forscher) und Objekt (Sinn) ganz neu zu bestimmen. Der Sinnforscher steht keinem Objekt gegenüber, das sich von außen beobachten und beschreiben ließe. Jede Beobachtung und jede Beschreibung verläuft im temporalen Medium des Sinns und vollzieht sich in dessen Kategorien. Wer ein Modell von Sinn nach Luhmannschen Kriterien bauen möchte, müsste dem Modell eine Komponente mitgeben, die das Modellbauen repräsentierte. Da diese Komponente sich selbst zu modellieren hätte, entstünde eine unendliche Regression, die Luhmann wohl als Musterfall von Rekursion betrachten würde.

Bei Luhmann, und den vielen Diskussionen um seine Theorie, wird das Verhaftetsein des Forschers in und von dem Medium, das er erforschen möchte, oft unter dem Stichwort Konstruktivismus abgehandelt. In unserem Kontext soll lediglich auf eine von dessen Implikationen aufmerksam gemacht werden. Welchen Wert der Konstruktivist den Ergebnissen seiner wissenschaftlichen Arbeit zugeschrieben wissen möchte, soll deshalb nicht in allen Einzelheiten diskutiert werden. Doch Eines scheint (mir) klar zu sein. Wie es auch mit der Wissenschaftlichkeit von solchen Studien stehen sollte, und was auch unter deren Wissenschaftlichkeit zu verstehen wäre, der Konstruktivist käme schwerlich auf die Idee für seine Befunde Entscheidbarkeit zu reklamieren. Ob Konstruktivisten diese Eigenschaft in jeder Wissenschaft für eine Chimäre halten sollten, braucht uns hier nicht zu kümmern. Die technologischen Erfolge der Naturwissenschaft, ermöglicht durch ihre bewährte Mischung von Mathematik und Experimenten, genügen an dieser Stelle um Entscheidbarkeit mit hinreichender Schärfe zu fassen. 
Textlinguisten mögen manches in Luhmanns Modellierung von Sinnereignissen finden, an dem kritisch anzusetzen wäre. Sie werden zu Recht beanstanden können, viele Details würden ungenau und nur mit oberflächlicher Fachkenntnis abgehandelt. Aber Luhmanns Einsicht in die gegenseitige Abhängigkeit von Forscher und Gegenstand bei Sinnstudien werden sie wenig entgegenzusetzen haben. Die Flucht der Strukturalisten in die Abstraktheit wird genau so wenig wie das sich Bescheiden in hermeneutische Praktiken die Lehre von Luhmann aufheben können. Wer die Kategorien des Sinns $\mathrm{zu}$ beobachten und $\mathrm{zu}$ beschreiben sucht, hat an einem temporalisierten Verfahren teil, für das es keine anderen Entscheidungsmöglichkeiten gibt als solche, die sich aus zukünftigen Sinnereignissen ergeben werden. Anders als die sinnfreie Entscheidung des Naturwissenschaftlers, die in einem Ja oder Nein terminiert, sind deren sinnhaften Entsprechungen unbegrenzte Laufzeiten beschert. Es gibt keinen Befund vom Sinnhaften, der selber, ein Sinnhaftes, nicht weiter zu prozessieren wäre.

\section{SCHLUSSBEMERKUNGEN}

Mit diesem Aufsatz bin ich der Frage nachgegangen, was von einer Textlinguistik zu erwarten wäre, die ohne die Kategorien von Sinn auszukommen suchte. Die Vorteile einer solchen Textlinguistik liegen auf der Hand. Sie wird als eine Wissenschaft einzurichten sein, die sich naturwissenschaftlichen Praktiken und deren erfolgsgewohnten Vorstellungen von Materialität und Determinismus weitgehend anschmiegen kann. Um dieser bisher wenig ausprobierten Variante eine erste Konkretisierung zu verschaffen, habe ich anfangs deren Konsequenzen mit Hilfe eines einfachen Modells zu verdeutlichen versucht.

Die Nachteile dieser Art von Textlinguistik sind nicht weniger offensichtlich. Was sie unter dem Titel von Textverwendung studiert, scheint wenig mit dem gemeinsam zu haben, was die Textverwender dabei zu erleben und zu tun meinen. Textverwendern kommt die Teilnahme an Textverwendungen selten als ein deterministisches Verfahren vor. Auch wären wenige aktive Textverwender mit einer Modellierung von ihrem Tun einverstanden, die das, was sich dabei ausspielte, als rein materielle Vorgänge repräsentierte. Textverwender neigen dazu sich und ihrem Tun einen beträchtlichen Freiraum zuzuschreiben (sicher größer bei Texterstellung als bei Textverstehen) und wären wohl auch schwerlich bereit, von ihren jeweiligen Erlebnissen (Erfahrungen) von Sinn als gleichgültigen Epiphänomenen abzusehen. Mit einem Wort: Eine solche Textlinguistik stieße im höchsten Maße gegen alles an, was eine normale Textintuition zu gebieten scheint.

In vielen Wissenschaften käme die Berufung auf Intuition einer wissenschaftlichen Kapitulation gleich. Auch ist nicht jeder Sprachdenker ihr gefolgt. Freges Modell von Vorstellungen und Sinn ist schwerlich intuitiv plausibel zu nennen. Sprechen ist eine grammatische Angelegenheit. Diese wird teils von psychischen, teils von logischen Komponenten begleitet. Die psychischen sind jedem in der Innerlichkeit seines Bewusstseins inkommunikabel versperrt, die logischen leben ihr ewiges Leben in einer nur sporadisch erfassbaren Zugänglichkeit. 
Karsten Hvidtfelt Nielsen, Braucht eine Textlinguistik Kategorien des Sinns?

Ein konsequenter Strukturalismus hätte wohl gleichfalls keine große Attraktivität für Normalintuitionen. Die Glossematik wäre da die Probe aufs Exempel. Ob viele Textverwender ihren Sinnerlebnissen Abstraktheit anmerken können, ist eher zweifelhaft. Coseriu scheint sich dessen bewusst gewesen zu sein. Seine Zuwendung zur Hermeneutik liest sich stellenweise als eine Reverenz gegen Sprach- und Textintuitionen, die ihm bei deren hermeneutischen Hütern besser aufgehoben scheinen. Hermeneutische Begriffe werden der Textlinguistik ohne Zweifel größere intuitive Plausibilität einbringen können, einen Zuwachs an Entscheidbarkeit wohl kaum. Das war auch Coserius Anliegen nicht.

Niemand wird von Luhmanns Theorien behaupten, sie seien intuitiven Erwartungen gegenüber besonders aufgeschlossen gewesen. Und doch gebührt aus den drei in diesem Aufsatz besprochenen Modellierungen von Sinn derjenigen von Luhmann die Ehre, den Zusammenhang von Sinn und Intuition am deutlichsten herausgebracht zu haben. Wo Frege mit seiner doppelten Verbannung von Vorstellung und Sinn die Intuition kurz und gut verabschiedete, und Coseriu seinen Sinn zwischen glossematischer Spekulation und hermeneutischer Intuition unentschieden schwanken ließ, lässt Luhmanns Modell von Sinn den Grund erkennen, weswegen Sinn und Intuition aufeinander angewiesen sind.

Wer Sinn erforschen will, muss Sinn selber prozessieren können. Wie dies geschieht, ist nach Luhmann den Prozessoren (Agenten) verborgen. Was ihnen dafür zur Verfügung steht, ist rekursive Teilnahme an temporalisierten Sinnprozessen. Diese Modellierung mag Textverwendern ebenso wenig einleuchten wie eine, die in Textverwendung nichts als deterministische Prozesse materieller Natur sehen will. Aber anders als bei dem MD-Modell, das mit dem Ausklammern des Sinn-Moments die Quelle intuitiven Befremdens ganz trockenlegt, kann man mit Hilfe von Luhmanns Modell erklären, weswegen es in ihm zu einem Schisma zwischen Modell und Intuition kommen muss. Wer Sinn als diejenigen systemproduzierten rekursiven Ereignisse erfahren könnte, die Sinn nach dem Modell zu sein hätte, müsste eine Position beziehen können, die das Modell kategorisch ausschließt. Nur von einem sinnfreien Außen beobachtet ließen sich Sinnereignisse in ihrer systemischen Eigentümlichkeit erkennen; in einem solchen Außen aber gibt es keine Beobachter.

So weit ich sehen kann, ist diese Paradoxie bei Luhmann durchaus eine gewollte, was selbstverständlich nicht heißen soll, sie sei gleichzeitig eine unumgängliche ${ }^{85}$. Also kommuniziert Luhmanns Modell den paradoxen Befund, dass ein systemtheoretisches Modell von Sinn, obwohl es mit interner Notwendigkeit gegen die mit Sinn verbundenen Intuitionen anstoßen muss, selbst die Sphäre des Sinnhaften nicht überschreiten kann. Luhmanns Modell ist daher nur insofern nicht intuitiv zu nennen, als es ex hypotheseos Sinn in dessen systemischer Beschaffenheit beschreibt. Selbst ist die Hypothese natürlich rein intuitiv und kann keine andere Beweiskraft für sich beanspruchen als die Zustimmung gleichgeschalteter Fachintuitionen.

Da bietet ein MD-Modell ganz andere Aussichten. Ein MD-Modell wird auch gegen Intuitionen anstoßen, aber nicht um alternative Fachintuitionen zu bedienen, die unter 
Karsten Hvidtfelt Nielsen, Braucht eine Textlinguistik Kategorien des Sinns?

paradoxen Bedingungen gedeihen. Was einem MD-Modell seine fehlenden Appelle an Textintuitionen beschert, ist gleichzeitig die Gewähr, dass man mit diesem Modell zu Ergebnissen kommen kann, über deren Bestand nicht Fachintuitionen, sondern Kriterien der Entscheidbarkeit richten können.

\section{LITERATUR}

Adamzik, Kirsten (2004), Textlinguistik. Eine einführende Darstellung, Tübingen: Niemeyer.

Antos, Gerd, Heike Tietz (Hgg.) (1997), Die Zukunft der Textlinguistik. Traditionen, Transformationen, Trends, Tübingen: Niemeyer.

Apel, Karl-Otto (1993), „Pragmatische Sprachphilosophie in transzendentalsemiotischer Begründung“, in: Stachowiak (1993), pp. 38 - 61.

Baker, G.P., P.M.S. Hacker (1984), Frege: Logical Excavations, Oxford: Oxford University Press.

Baker, G.P., P.M.S. Hacker (1987), "Dummett's Dig: Looking-Glass Archaeology", The Philosophical Quarterly, 37 (1987), pp. 86 - 99.

Barthes, Roland (1988), Das semiologische Abenteuer, Frankfurt/M.: Suhrkamp.

Becker, Karl Ferdinand (1841[1970]), Organism der Sprache, Hildesheim: Georg Olms.

Brinker, Klaus (19974), Linguistische Textanalyse. Eine Einführung in Grundbegriffe und Methoden, Berlin: Erich Schmidt.

Brinker, Klaus et al. (Hgg.) (2000), Text- und Gesprächslinguistik (= Handbücher zur Sprach- und Kommunikationswissenschaft, Bd. 16.1), Berlin: de Gruyter.

Brinkmann, Hennig (1980), Mittelalterliche Hermeneutik, Tübingen: Niemeyer.

Bühler, Karl (1927[1978]), Die Krise der Psychologie, Frankfurt/M.: Ullstein.

Bühler, Karl (1934[1965]), Sprachtheorie, Stuttgart: Fischer.

Chalmers, David J. (1996), The Conscious Mind. In Search of a Fundamental Theory, Oxford: Oxford University Press.

Chomsky, Noam (1959), "Review of Skinner, Verbal Behavior", Journal of the Linguistic Society of America, 35 (1959), pp. 26 - 58.

Chomsky, Noam (1969), "Quine's Empirical Assumptions", in: Davidson/Hintikka (1969), pp. 53 - 68.

Coseriu, Eugenio (1981), Textlinguistik. Eine Einführung, Tübingen: Günter Narr.

Coseriu, Eugenio $\left(2003^{2}\right)$, Geschichte der Sprachphilosophie von den Anfängen bis Rousseau, Tübingen: Francke.

van Dalen, Dirk (1983), "Algorithms and Decision Problems. A Crash Course in Recursion Theory", in: Gabbay/Gunther (1983), pp. 409 - 478.

Dascal, M. et al. (Hgg.) (1992), Sprachphilosophie (= Handbücher zur Sprach- und Kommunikationswissenschaft, Bd. 7.1), Berlin: de Gruyter.

Davidson, Donald, Jaako Hintikka (eds.) (1969), Words and Objections. Essays on the Work of W.V. Quine, Dordrecht: D. Reidel.

Davidson, Donald (1980), Essays on Actions and Events, Oxford: Clarendon Press.

Dennett, Daniel C. (1991), Consciousness Explained, Harmondsworth: Little, Brown \& Co.

van Dijk, Teun A. (1980), Textwissenschaft, Tübingen: Niemeyer. 
Karsten Hvidtfelt Nielsen, Braucht eine Textlinguistik Kategorien des Sinns?

Dummett, Michael (1981), The Interpretation of Frege's Philosophy, London: Duchworth.

Dummett, Michael (1984), "The Unsuccessful Dig", in: Wright (1984), pp. 194 - 226.

Feikle, Helmuth (2000), "Die pragmatische Wende in der Textlinguistik", in: Brinker et al. (2000), pp. 64 - 82 .

Frege, Gottlob (1884[1987]), Grundlagen der Arithmetik, Stuttgart: Suhrkamp.

Frege, Gottlob (1962), Funktion, Begriff, Bedeutung, Göttingen: Vandenhoeck \& Ruprecht.

Frege, Gottlob (1966), Logische Untersuchungen, Göttingen: Vandenhoeck \& Ruprecht.

Frege, Gottlob $\left(1983^{2}\right)$, Nachgelassene Schriften, Hamburg: Felix Meiner.

Gabbay, D., F. Gunther (eds.) (1983), Handbook of Philosophical Logic, Dordrecht: D. Reidel.

Hartung, Wolfdietrich (1997), “Text und Perspektive. Elemente einer konstruktivistischen Textauffassung“, in: Antos/Tietz (1997), pp. 13 - 25.

Haselbach, Gerhard (1966), Grammatik und Sprachstruktur. Karl Ferdinand Beckers Beitrag zur Allgemeinen Sprachwissenschaft in historischer und systematischer Sicht, Berlin: de Gruyter.

Hjelmslev, Louis (1966), Omkring sprogteoriens grundloeggelse, København: Akademisk Forlag.

Kneer, Georg (1997), "Beobachten, Verstehen und Verständigung", in: Sutter (1997), pp. $50-69$.

Knobloch, Clemens (1988), Geschichte der psychologischen Sprachauffassung in Deutschland von 1850 bis 1920, Tübingen: Niemeyer.

Knobloch, Clemens (1992), "Wilhelm Wundt", in: Dascal et al. (1992), pp. 412 - 431.

Luhmann, Niklas (1980[1993]), Gesellschaftsstruktur und Semantik. Studien zur Wissenssoziologie der modernen Gesellschaft, Bd. 1, Frankfurt/M.: Suhrkamp.

Luhmann, Niklas (1998), Die Gesellschaft der Gesellschaft, 2 Bde., Frankfurt/M.: Suhrkamp.

Morris, Charles (1946), Signs, Language and Behavior, New York: Prentice-Hall.

Nielsen, K. Hvidtfelt (1982), "A Formal Investigation of Five Glossematic 'Functions'”, Acta Linguistica Hafniensia, 17 (1982), pp. 131 - 138.

Nielsen, K. Hvidtfelt (2002), „Cicero und Wittgenstein. Zur Verortung des rhetorischen Sprechens", Rhetorik. Ein internationales Jahrbuch, 21 (2002), pp. 102 - 118.

Nielsen, K. Hvidtfelt (2003), Interpreting Spinoza's Arguments. Toward a Formal Theory of Consistent Language Scepticism, Lewiston: Mellen.

Paul, Hermann (1920[1975]), Prinzipien der Sprachgeschichte, Tübingen: Niemeyer.

Putnam, Hilary (1960[1975]), "Minds and Machines", in: Putnam (1975), pp. 362 - 385.

Putnam, Hilary (1975), Mind, Language and Reality (= Philosophical Papers, vol. 2), Cambridge: Cambridge University Press.

Quine, Willard Van Orman (1960), Word and Object, Cambridge/Mass.: John Wiley.

Quine, Willard Van Orman (1969), "Reply to Chomsky", in: Davidson/Hintikka (1969), pp. 302 - 311.

Saussure, Ferdinand de (1915[1972]), Cours de linguistique générale, Paris: Payot.

Scherner, Maximilian (1984), Sprache als Text. Ansätze zu einer sprachwissenschaftlich begründeten Theorie des Textverstehens, Tübingen: Niemeyer. 
Scherner, Maximilian (2000), „Kognitionswissenschaftliche Methoden in der Textanalyse“, in: Brinker et al. (2000), pp. 186 - 195.

Skinner, B.F. (1957), Verbal Behavior, New York: Prentice-Hall.

Skorupski, John (1984), “Dummett's Frege”, in: Wright (1984), pp. 227 - 243.

Sluga, Hans (1980), Gottlob Frege, London: Routledge \& Kegan.

Spinoza, Baruch de (1677[1925]), Ethica. Ordine geometrico demonstrata, Heidelberg: Carl Winters.

Stachowiak, Herbert (Hg.) (1993), Sprachphilosophie, Sprachpragmatik und formative Pragmatik (= Pragmatik. Handbuch pragmatischen Denkens, Bd. IV), Hamburg: Felix Meiner.

Steinthal, H. (1863[1961]), Geschichte der Sprachwissenschaft bei den Griechen und Römern mit besonderer Berücksichtigung der Logik, 2 Bde., Bonn: Ferd. Dümmler.

Sutter, Tilmann (Hg.) (1997), Beobachtung verstehen, Verstehen beobachten. Perspektive einer konstruktivistischen Hermeneutik, Opladen: Westdeutscher Verlag.

Weinrich, Harald (1993), Textgrammatik der deutschen Sprache, Mannheim: Dudenverlag.

Wittgenstein, Ludwig (1971[1958]), Philosophische Untersuchungen, Frankfurt/M.: Suhrkamp.

Wright, Crispin (ed.) (1984), Frege: Tradition and Influence, Oxford: Blackwell.

\section{ANMERKUNGEN}

1 Einen guten Überblick über diese Diversität bietet Brinker et al. (2000).

2 Für die in meinem Buch Nielsen (2003) verwendete Sprachlogik zeichnet eher die sprachskeptische Sorte von Quine und Kripke als die Tradition von Montague.

3 Siehe Nielsen (2003: 152). Der epistemische Monismus lässt sich als eine von Quine inspirierte Weiterentwicklung und Verschärfung von dem in Davidson (1980) vorgeschlagenen anomalen Monismus verstehen. Als eine zweite Inspiration für den epistemischen Monismus wäre Wittgenstein (1971[1958]) anzuführen. Vgl. z. B. § 306: "Warum soll ich denn leugnen, dass ein geistiger Vorgang da ist?!“. Wie Wittgenstein die Existenz geistiger Ereignisse natürlich nicht leugnen will, nur deren Relevanz für die philosophische Beschreibung, so besteht der epistemische Monist nur darauf, dass mentale Annahmen in der wissenschaftlichen Forschung überflüssig sind. Dass sie in anderer Hinsicht von Nutzen (und im höchsten Maße sinnvoll) sein können, soll selbstverständlich nicht bezweifelt werden.

4 Es handelt sich natürlich um die berühmte Proposition 5,3 aus Ethica: „Affectus, qui passio est, desinit esse passio, simulatque ejus claram, \& distinctam formamus ideam." (Spinoza 1677[1925]: 282). Versteht man unter passio ein inneres Erlebnis, das als Affekt keine Erkenntnis sein kann, und unter dessen clara, \& distincta idea diejenige wissenschaftliche Erkenntnis, die den Affekt auf seinen wahren Grund zurückführt und damit aufhebt, besagt die Proposition, dass die Wissenschaft in dem Maße, als ihr sichere Erkenntnisse zuwachsen, alle inneren Erlebnisse, die nicht selber solche Erkenntnisse sind, auflösen wird. Argumentation für diese Interpretation findet sich in Nielsen (2003).

5 Es muss sicher nicht extra gesagt werden, dass Spinoza auch für mein Bekenntnis zu Determinismus Pate gestanden hat.

6 Für den interessierten Laien: Selbst die Quantenmechanik verfährt strikt deterministisch; nur beim Übergang von mikroskopischen zu makroskopischen Ereignisstrukturen tritt ein stochastisches Element auf, das freilich mathematisch streng geregelt ist. In Nielsen (2003) wird die Relevanz quantenmechanischer Betrachtungen für die Bewusstseinsforschung kurz 
diskutiert (Nielsen 2003: 51, 55, 121, 124-128, 135).

7 Siehe z. B. die Debatte um Chalmers (1996) (in der Zeitschrift Journal of Consciousness Studies 3f. (1996f.)).

8 Die Psychologie des 19. Jahrhunderts war von dem Herbartismus und dessen deterministischem Denken geprägt. Zu dieser Tradition siehe Knobloch (1988).

9 Das soll nicht heißen, dass von Determination in der Textlinguistik nie die Rede gewesen ist. In Weinrich (1993) z. B. wird die Grundsituation des Sprechens als eine Dialektik von Anweisung und Freiheit charakterisiert, wobei unter Anweisung determinierende Faktoren wie Kontext, Situation und - vor allem - die Determinationsgefüge der Sprachzeichen gemeint sind (Weinrich 1993, 18, 21). Was mit Freiheit bezeichnet werden soll, scheint Weinrich eine Selbstverständlichkeit gewesen zu sein. Eben dies soll mit der hier präsentierten These bezweifelt werden.

10 Oder, wenn man will, die Falsifikation (Popper).

11 Die Diskussion um Entscheidbarkeit entstand nach Gödels epochalem Befund im Jahre 1931. Gödel zeigte, dass jede nur mäßig ausgebaute formale Sprache (wie z. B. die Peano-Arithmetik) wahre Sätze enthalten würde, die weder als Thesen zu beweisen noch als deren Negationen abzulehnen wären. Zu einer ausführlichen Diskussion von Gödels Unentscheidbarkeitsbeweis und dessen Bedeutung für die Entscheidbarkeit wissenschaftlicher Sprachen siehe Nielsen (2003).

12 Sieht man von der Phonetik ab, lässt sich diese Aussage wohl auch für die gesamte Linguistik generalisieren.

13 Siehe z. B. Morris (1946), Skinner (1957), Putnam (1960[1975]), Dennett (1991). Bemerkt, dass Putnam in späteren Werken von der hier angesprochenen Position abrückte.

14 Chomsky (1959) wird noch in vielen Einführungen in sprachwissenschaftliche Einzeldisziplinen als die definitive Disqualifizierung des Sprachbehaviorismus zitiert. Dass aber Chomskys Verriss von nur mäßiger Kenntnis des Buches zeugte, über das er so gnadenlos urteilte, ist mehrmals nachgewiesen worden. Zu dieser Diskussion siehe Nielsen (2002) (mit weiterführender Literatur).

15 Die sind natürlich wiederum in die umfassenderen Prozesse der Sozialisation integriert. In diesem Modell soll aber von beiden erwähnten Prozesstypen, Spracherwerb und Sozialisation, abgesehen werden.

16 In der behavioristischen Tradition werden Erfolgskriterien unter dem Begriff von reinforcement diskutiert. Siehe Skinner (1957: 29ff.).

17 Nur aus Gründen der Einfachheit werden Zustände als atomare Einheiten betrachtet. Über deren etwaige Module weiter zu spekulieren erübrigt sich in diesem Zusammenhang.

18 Auch Maschinen werden nach entsprechender Konditionierung koordiniertes Verhalten aufweisen.

19 Der epistemische Monist ist solcher Sorgen enthoben. Vgl. Wittgenstein (1971[1958]: § 304): Der imaginierte Gegner von Wittgensteins Reduktionismus führt dessen Vertreter vor. ,'Und doch gelangst du immer wieder zum Ergebnis, die Empfindung selbst sei ein Nichts'“. „Nicht doch.“, antwortet der Vertreter, "Sie ist kein Etwas, aber auch nicht ein Nichts.“. Genau so möchte der epistemische Monist demjenigen antworten, der für ein nicht beobachtbares Etwas (im makroskopischen Bereich!) eine über- oder nebenphysische Realität reklamieren wollte.

20 Siehe Coseriu $\left(2003^{2}\right)$ oder den seinem Alter zum Trotz noch sehr empfehlenswerten Steinthal (1863[1961]).

21 Zum Terminus des Sprachbildes und zur Diskussion des Begriffes, der mit diesem Terminus gemeint sein soll, siehe Nielsen (2002).

22 Siehe Morris (1946), Skinner (1957), Quine (1960).

$23 \mathrm{Zu}$ einer Gesamtdarstellung des Schriftsinnes im Mittelalter siehe Brinkmann (1980). 
24 Als Beispiele wären die Debatten zwischen Baker/Hacker und Dummett (Auszüge davon in Baker/Hacker 1984 und 1987 und in Dummett 1984), oder die zwischen Sluga und Dummett (Auszüge davon in Sluga 1980 und Dummett 1981) zu erwähnen.

25 Bei Frege müsste man eigentlich von Satzsinn sprechen, aber hier soll von diesem - für Textlinguisten sonst so wichtigen - Unterschied abgesehen werden.

26 Vgl. Bühler (1927[1978]: 62): „Ähnlich wie Meinong [...] hat auch Frege der Sache nach eine Zweiheit im Sprachsinn erkannt (Frege spricht von Sinn und Bedeutung)“.

27 Sprachvorstellungen werden bei Frege oft als Färbung, Beleuchtung oder gar Dichtung umschrieben.

28 Siehe Knobloch (1988).

29 Siehe Frege (1884[1987]: 19f.).

30 In Frege (1962: 40-65), zitiert als (SB, Seitenzahl).

31 In Frege (1966: 30-53), zitiert als (G, Seitenzahl).

32 Würden wir Bedeutung als Relation modellieren, kämen wir auf eine Lehre vom drei-fachen Sinn - ersteres ist wohl eher nicht Freges Intention.

33 Mit dem Begriff der Vorstellung und seiner Modellierung als ein privates Erlebnis folgt Frege demjenigem Zweig der Sprachpsychologie, der unter anderen in Paul (1920[1975]) entfaltet werden sollte.

34 Siehe z. B. Skorupski (1984) für eine instruktive und leicht zugängliche Übersicht über Dummetts Auffassung (weniger klar bei dem Urheber selbst in Dummett 1981) oder Baker/ Hacker (1984) für eine zwar nicht besonders einflussreiche, dafür aber nüchterne Interpretation vom Phänomen Sinn bei Frege.

35 Auch diese Frage ist in der Fregeforschung kontrovers, u.a. wegen der vielen nominalistischen Einschläge bei Frege.

36 Siehe $(\mathrm{G}, 33)$.

37 Siehe die berühmte zweite Note über Aristoteles in (SB, 42) und die Diskussion über Dr. Lauben in $(G, 38-40)$.

38 Zitat aus „Erkenntnisquellen der Mathematik und der mathematischen Naturwissenschaften“, in Frege (19832: 286-294, 288).

39 Nicht nur Frege spekulierte im 19. Jahrhundert über das Verhältnis zwischen Sprache und Logik nach. Die so genannte rationale Grammatik erreichte in der ersten Hälfte des Jahrhunderts durch die Werke von Karl Ferdinand Becker (siehe Becker 1841[1970]) große Popularität, nicht zum wenigsten in den Schulgrammatiken. Dazu siehe Haselbach (1966).

40 Beispiele aus Freges Werken sind Legion.

41 Zitat aus Quine (1969: 303), wo Quine versucht einige von den vielen Missverständnissen, die Chomsky (1969), Chomskys Rezension von Quine (1960), entstellten, zu korrigieren; mit wenig Erfolg, wie aus Chomskys späteren Werken erhellt. Zu dieser Diskussion (mit weiterführender Literatur) siehe Nielsen (2003).

42 So z. B. in seinen frühen Aufzeichnungen zur Logik, in Frege $\left(1983^{2}: 1-8,2\right)$.

43 Aufzeichnungen zur Logik, in Frege $\left(1983^{2}: 1-8,2\right)$.

44 In Coseriu (1981: 51); im Text wird Coseriu (1981) als (TL, Seitenzahl) zitiert.

45 Vgl. Nielsen (1982)

46 Vgl. Feikle (2000). Das beherrschte Glück Coserius in der Forschung scheint der Verbreitung seiner Textlinguistik keinen Abbruch getan zu haben, die im Jahre 1994 ihre dritte Auflage verbuchen konnte.

47 Schon Scherner (1984), ein für diese Entwicklung richtungsweisendes Buch, hat eine, ob 
auch im Wesentlichen kritische, Besprechung von Coseriu (157-58, 160-161). Freundlicher ist Adamzik (2004), die bei Coseriu Stütze für ihre Stilistik findet (146f.). Adamzik hätte auch ihre Betonung der hermeneutischen Aufgabe der Textlinguistik $(15-16,61)$ durchaus auf Coseriu zurückführen können.

Vgl. Coseriu $\left(2003^{2}\right)$.

Siehe z. B. Barthes (1988).

50 Unten wird noch eine dritte hinzukommen.

51 Strukturalisten würden an dieser Stelle kontern wollen, sie hätten Fragen nach dem Wo mit solchen nach dem Wie ersetzt. Aber auch ein Wie braucht Realität, braucht ein Wo, um sich ereignen zu können.

52 Was (TL, 31) Sprachfunktionen hieß, soll vermutlich mit dem, was (TL, 47) einzelsprachliche Funktionen heißt, identifiziert werden. Bezeichnungsfunktionen kommen dann extra hinzu.

53 Bei der Verwendung von Termini wie Bedeutung und Bezeichnung scheint Coseriu, absichtlich oder nicht, die Unterscheidung zwischen nomina actionis und nomina acti nicht beachtet zu haben.

54 In seiner Sprachtheorie sah Hjelmslev die Möglichkeit einer unendlichen Hierarchie von Sprachen voraus. Jede Sprache besteht nach Hjelmslev aus einer Inhaltsseite und eine Ausdrucksseite. Auf einer ersten Stufe bilden keine dieser Seiten selber eine Sprache. Auf der zweiten Stufe der Hierarchie besteht entweder die Ausdrucksseite oder die Inhaltsseite (im exklusiven Sinne) selber aus Sprachen. Und generell: unter einer Sprache der n'ten Stufe (n > 1) ist eine Sprache zu verstehen, deren Inhaltsseite oder Ausdrucksseite eine Sprache der n-1'sten Stufe ausmacht. Besteht die Inhaltsseite einer n-stufigen Sprache selber aus Sprache, haben wir es nach Hjelmslev mit einer Metasprache zu tun; anderenfalls mit einer Konnotationssprache (Hjelmslev, 1966: 101f.).

55 Vgl. die noch heute aktuelle Diskussion in Bühler (1927[1978]). Mit dem Hinweis auf die Unentbehrlichkeit der Sinndimension für die Linguistik ist für Bühler auch die Grenze gezogen worden zwischen dem, was ein Behaviorismus leisten kann, und dem, wofür man eine Sprachpsychologie benötigt.

56 Dies ist übrigens bei Bühler nicht anders: Auch er kennt die forschungstrategische Nötigung Formalisierungen, wie er seine Abstraktionen nennt, vorzunehmen (Bühler 1934[1965]: 49-50); dass aber solche eben nur Zurechtstutzungen der konkreten Sprachereignisse sind, von denen jeder Sprachforscher auszugehen hat, war ihm völlig klar.

57 Bekannt ist das Schwanken Saussures zwischen einer psychischen und einer abstrakten Existenzweise des Zeichens.

58 van Dijk (1980: 51) sieht die Möglichkeit voraus, seine „Regeln rein automatisch anwenden“ zu können, also „algorhythmisch [sic! Ist ein Wortspiel auf Rhythmus intendiert?] z. B. mit Hilfe eines Computers“. Doch kommt van Dijk (1980) nirgendwo auch nur in die Nähe von einer Bestimmtheit, die eine faktische Algor[h]i[y]thmisierung seiner Makroregeln gestatten würde.

59 Vgl. Kneer (1997).

60 In Luhmann (1980[1993]), im Text zitiert als (GS, Seitenzahl), verweist Luhmann auf die Interdisziplinarität seiner Studien und zählt unter den Disziplinen, in die sein Thema eingreift, auch die Textlinguistik hinzu (GS, 17). Konkrete Verweise auf (mir bekannte) textlinguistische Arbeiten habe ich zwar bei Luhmann nicht ausmachen können. Auch scheinen wenige Textlinguisten Luhmanns Invitation gefolgt zu sein. Eine Ausnahme ist Scherner (2000), der eine gute, wenn auch kurze Darstellung davon bietet, wie Luhmanns systemtheoretischer Konstruktivismus mit einer kognitionswissenschaftlichen Textanalyse zu verbinden ist. Scherner identizifiert als „das eigentliche Basisproblem“ der Kognitionsforschung "die Geschlossenheit des individuellen Bewusstseins" (192), die „Kommunikation unwahrscheinlich“ (193) (vgl. GG, 190-191) (siehe Anmerkung 61) macht, und hebt hervor, dass Luhmanns Theorie die Ehre gebührt, sich diesem Problem in aller Radikalität gestellt zu haben. In Hartung (1997), der zwar keinen direkten Verweis auf Luhmann enthält, wird der 
theoretische Rahmen für eine konstruktivistische Textlinguistik skizziert.

61 Zitiert (GG, Seitenzahl). Gelegentlich soll auch, wie schon in Anmerkung 60, auf Luhmann (1980 [93]) hingewiesen werden.

62 Darüber hinaus scheint Luhmann sich auch mit Hallidays funktionaler Linguistik beschäftigt zu haben (GG, 38).

63 Als Urheber dieser Bestimmung von Form gilt nach Luhmann der Mathematiker George Spencer Brown (GG, 60).

64 Dieser Vergleich zwischen Luhmann und Hjelmslev gilt mit einer, zwar wesentlichen, Modifikation, die sich aus der Universalität des Sinns bei Luhmann ergeben wird.

65 So ganz nach der 'Definition' von Form: "Eine Form ist letztlich eine Unterscheidung, die in sich selbst als Unterschiedenes widervorkommt." (GG, 57)

66 In einer Note (9) versucht Luhmann - vielleicht - auf diese Frage einzugehen.

67 Luhmann verweist auf den Mathematiker Brown (GG, 45), hat auch zu Gödels Gebrauch von Rekursionen einen Kommentar (GG, 68). Dennoch verharren seine Thematisierungen von Rekursion durchaus im Informellen (GG, 68).

68 Siehe van Dalen (1983).

69 Mit einem Wort aus Browns Laws of Form wird bei Luhmann diese rekursive Sinnstruktur auch re-entry genannt.

70 Luhmann widmet diesen Fragen unter dem Leitbegriff Autopoiesis (Maturana) ein längeres Kapitel in Luhmann (1998).

71 Dabei wäre das Gleichheitszeichen zu temporalisierien, etwa wie in der Informatik mit , =:'.

72 Vielleicht besonders deutlich in Kapitel 1 von Luhmann (1980[1993]), z. B. (GS, 19).

73 Diese Markierung wird bei Luhmann durchgängig Bezeichnung oder Referenz genannt, da aber diese Termini sprachtheoretisch ,vorbelastet' sind, ziehe ich die neutralere Markierung vor.

74 Strukturalisten dürfte das Akzept von potenziellen Größen keine großen Schwierigkeiten bereiten. Wer z. B. die Inhaltsform (um glossematische Terminologie zu wählen) studiert, wird die Ausdrucksform als latente, nicht aktualisierte Seite des Zeichens, betrachten können.

75 Der Übergang von einer Herbartischen Psychologie zu einer Brentanoschen am Ende des 19. Jahrhunderts führte u.a. zu einer fachlichen Legitimierung der Introspektion als Mittel zur Gewinnung von Erkenntnissen über das Innenleben. Der Kritik, die der (in dieser Hinsicht) Herbartische Wundt an den introspektiven Praktiken des Brentanisten Marty übte, müsste Luhmann ohne weiteres sich anschließen können (vgl. Anmerkung 60). Zu der Wundt-Marty Debatte siehe Knobloch (1992).

76 Der Grund, weswegen diese Formulierung für Luhmann wichtig ist, erhellt aus der beigefügten Note (56): „Das schließt im übrigen diese Aussage selbst ein.“

77 Eine Formulierung, auf die ich Wert lege.

78 Die müsste man dann anders ausfüllen, etwa wie Frege mit logischem Sinn.

79 So z. B. in Luhmann (1980[1993]), wo auch folgende Definition von Semantik vorkommt: „Unter Semantik verstehen wir [...] einen höherstufig generalisierten, relativ situationsabhängig verfügbaren Sinn.“ (GS, 19)

80 Auf Saussures Unentschlossenheit ist schon in Anmerkung 57 hingewiesen worden.

81 Luhmann widmet der Schrift und dem Text als eigenen Medien eine Aufmerksamkeit, die die Textlinguisten interessieren müsste. Obwohl er sich hauptsächlich mit der Genese der Schrift beschäftigt, werfen viele Kommentare ein neues Licht auf die Transformationen, die der Übergang von gesprochener zu geschriebener Sprache der Kommunikation aufnötigte (GG, 249-290).

82 Siehe Apel (1993), wobei bemerkt werden soll, dass der Hinweis auf Apriorizität konträre 
Karsten Hvidtfelt Nielsen, Braucht eine Textlinguistik Kategorien des Sinns?

Dienste leistet: bei Apel um jede Sprach- und Sinnskepsis zu unterbinden, bei Luhmann um deren Unvermeidlichkeit zu begründen.

83 Vgl. Anmerkung 6 zur Quantenmechanik. Bemerkt, dass die Unschärferelation mathematisch gesehen ein äußerst Scharfes ist.

84 Luhmann spricht in solchen Fällen von interner Umwelt.

85 Mit seinen Begriffen von Beobachtung erster und zweiter Ordnung will Luhmann diese Paradoxie nicht aufgelöst, sondern operationalisierbar gemacht haben. Beobachtungen erster Ordnung bilden das Objekt von Beobachtungen zweiter Ordnung. Luhmanns Theorie ist eine Reflexion zweiter Ordnung, deren Komplizität mit den Objekten erster Ordnung nach Luhmann unaufhebbar ist. Kein Zweifel, dass Selbstreflexivität mit Paradoxien eine Koexistenz führt. Diese Einsicht hat schon Cantors Antinomien beflügelt. Aber daraus, wie Luhmann, schließen zu wollen, Paradoxie sei eine notwendige (und erwünschte) Eigenschaft von selbstreflexiven Theorien, heißt eigene Neigungen vorschnell verabsolutieren zu wollen. Wie man selbstreflexive Sprachskepsis auf konsistente, heißt nicht-paradoxe, Art sowohl ausüben als auch formalisieren kann, habe ich mit Nielsen (2003) demonstriert. 